\title{
Structural determinants of substrate recognition and catalysis by heparan sulfate sulfotransferases
}

Tarsis Ferreira Gesteira ${ }^{\S *}$, Tainah Dorina Marforio ${ }^{\ddagger}$, Jonathan Wolf Mueller ${ }^{\dagger}$, Matteo Calvaresi ${ }^{\ddagger}$ and Vivien Jane Coulson-Thomas ${ }^{\S}$

§College of Optometry, University of Houston, Houston, Texas 77004, United States

† Dipartimento di Chimica “Giacomo Ciamician”, Università di Bologna, Bologna 40126, Italy

$\dagger$ Institute of Metabolism and Systems Research, University of Birmingham, Birmingham B15 2TQ, UK.

*tgferrei@central.uh.edu

Table of Contents

Supporting Methods

System Setup and Molecular Dynamics Simulation

Molecular Mechanics Generalized-Born Surface Area (MM-GBSA)

Multiple sequence alignment of the catalytic domains of eight HS2STs

Enzyme assays

Metadynamic simulations (sugar puckering and water coordination)

QM/MM setup and ONIOM calculations

Table S1

Ring puckering analysis

Interaction fingerprint analysis

Grid Inhomogeneous solvation analysis

Figure S1. Multiple sequence alignment of HS2ST from different species

Table S1. Activation barriers obtained by DLPNO-CCSD(T)/cc-pVTZ - cc-pvTZ

Figure S2. Free energy surface (FES) obtained from HS2ST: ${ }^{1} \mathrm{C}_{4}$-IdoA MD simulation

Figure S3. Cross correlation and dihedral PCA analysis

Figure S4. Root mean square deviations conformations (RMSD) for HS2STs

Discussion S1

Figure S5. MM-GBSA over time $(2 \mu \mathrm{s})$ as calculated for HS2ST conditions studied

Table S2. MM-GBSA computed binding free energies $(\Delta \mathrm{G})$ for HS2ST complexes formation

Table S3. Per-residue MM-GBSA computed binding free energies $(\Delta \mathrm{G})$ for HS2ST complexes formation

Figure S6. HS2ST Mutants analysis

Figure S7. Puckering metadynamics

Figure S8. Uronic acid epimers binding to HS2ST

Figure S9. 3D-representation of the critical points obtained at the ONIOM[M06-2X/6-31+G*:ff14SB] level of theory for the catalytic mechanism of the sulfate transfer from PAPS to the IdoA acceptor in different conformations

Figure S10. 3D-representation of the critical points obtained at the ONIOM[M06-2X/6-31+G*:ff14SB] level of theory for the catalytic mechanism of the sulfate transfer from PAPS to ${ }^{4} \mathrm{C}_{1}-\mathrm{GlcA}$ and ${ }^{1} \mathrm{C}_{4}-\mathrm{GlcA}$

Discussion S2

Figure S11. Molecular dynamics analysis of HS6ST

Discussion $\mathrm{S3}$

Table S4. MM-GBSA computed binding free energies $(\Delta \mathrm{G})$ for HS6ST complexes formation

Figure S12. MM-GBSA as calculated for HS6ST with IdoA $2 \mathrm{~S}_{+4} / \mathrm{GlcA}_{+4} / \mathrm{IdoA}_{+4} / \mathrm{GlcA} 2 \mathrm{~S}_{+4}$ simulations

Figure S13. Radial distribution of water around the catalytic histidine and pairwise decomposition between GlcNS and W158

Figure S14. HS6ST metadynamics analysis

Figure S15. Cooperative motions underlying the functional dynamics in the apoprotein and sugar bound HS6STs

Figure S16. . HS6ST substrate at position +4 influences HS6ST motility and active site hydration 


\section{Discussion S4}

Figure S17. Interaction fingerprinting for HS6ST simulations

Figure S18. Contact analysis for HS6ST simulations

Discussion S5

Figure S19. 3D-representation of the critical points obtained at the ONIOM[M06-2X/6-31+G*:ff14SB] level of theory for the catalytic mechanism of the sulfate transfer from PAPS to GlcNS +1 for HS6ST:GlcA +4

Discussion S6

Figure S20. Enzyme-ligand fingerprinting for HS6ST:Ido2 $\mathrm{S}_{+4}$ towards non-reducing end extensions

Figure S21. The electrostatic surface potential (generated with APBS ${ }^{1}$ ) of HS2ST and HS6ST

References

\section{Supplemental Methods}

System Setup and Molecular Dynamics Simulation. PAPS was parameterized based on the crystal structure of human bile salt sulfotransferase SULT2A1 (pdb code 4IFB). Briefly, we calculated the partial atomic charges using the Restrained Electrostatic Potential method (RESP) at the HF/6-31G(d) level of theory. Then, using the antechamber module, the corresponding parameters were generated by means of the parmchk module of Amber16. Standard amino acid and carbohydrate residues were parameterized with the FF12SB and GLYCAM06 (J-1) force fields, respectively. Glucuronic and iduronic isomers were modeled using glycam-web ${ }^{2}$.

The crystal structure of HS2ST (pdb ID 4NDZ) presents the active site at the homotrimer interface ${ }^{3}$. Here, the substrate engages hydrogen bond network with polarizable sidechains of amino acid residues present in the catalytic task, as highlighted in Figure 1 and discussed in recent work ${ }^{3,4}$. The complete protein complexed with PAPS and hexasaccharide was inserted into a rectangular box $(130 \AA x 130 \AA x 106 \AA)$ of pre-equilibrated TIP3P water molecules ${ }^{5}$ and $\mathrm{Na}^{+}$counter ions to neutralize the total charge. The protonation states of ionizable residues were predicted by the $\mathrm{H}^{++}$web server http://biophysics.cs.vt.edu/ $\mathrm{H}++^{6}$ and further compared with the available experimental information. The resulting system with PAPS and hexasaccharide within the 2-OST active sites was minimized performing three molecular mechanics (MM) minimization steps by applying the steepest descent and conjugate gradient methods.

The initial stage includes the imposition of harmonic restraints on the hexasaccharide substrate, the enzyme and PAPS, while waters and ions are kept unrestrained. For a second minimization, just the PAPS and hexasaccharide were restrained. For the last minimization step, only the protein side chains were restrained, with the remaining of the system free. The system is then subjected to molecular dynamics (MD) simulation under periodic boundary conditions (PBC), using a cutoff of $10 \AA$ for all Lennard-Jones and electrostatic interactions and the particle-mesh Ewald method to treat long-range electrostatic effects. The Michaelis complex has been gradually heated from 0 to $300 \mathrm{~K}$ within a closed system with constant volume, with an increment of $30 \mathrm{~K}$ during 10 steps of 20 ps each, imposing a harmonic restrain (5 kcal mol-1 $\AA$-2) on both PAPS and hexasaccharide using Langevin dynamics ${ }^{7}$. A simulation of the system is then performed under the NPT ensemble to ensure periodic box uniformity and control its density. After reaching $300 \mathrm{~K}$, we carried out four incremental steps of 10 ps each, at $1 \mathrm{~atm}$ of pressure, with a weak restraint on the side chains of the protein residues, employing an isotropic weakcoupling algorithm and the Berendsen barostat ${ }^{8}$ and then one more step of $160 \mathrm{ps}$ with the same restrain up to reach a density around $1 \mathrm{~g} \mathrm{~cm}^{-3}$. Finally, starting from the last configuration, an equilibration step of $10 \mathrm{~ns}$ and a production of $2 \mu \mathrm{s}$ have been calculated at $300 \mathrm{~K}$ under the NVT ensemble without any restraints apart from employing the SHAKE algorithm ${ }^{9,10}$ to constrain all the covalent bonds containing hydrogen. The MD simulation was carried out using the AMBER 16 GPU (CUDA) version of the PMEMD package ${ }^{11,12}$, with restart files being generated every $250 \mathrm{~ns}$. As the 2OST is a homotrimer one MD was performed and the triplicate results represent each monomer analysis. The crystal structure of HS6ST at both

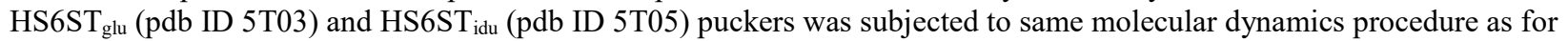
HS2ST, with the enzyme being inserted into a rectangular box with dimensions $90 \AA x 94 \AA x 96 \AA$.

Molecular Mechanics Generalized-Born Surface Area (MM-GBSA). Molecular mechanics Generalized Born surface area (MM-GBSA) methods ${ }^{13-15}$ implemented in Amber 16.0 software ${ }^{16}$ package were applied to compute the binding free energy for the protein-ligand complex. For each system, the key coordinates were extracted from equilibrium trajectories every $100 \mathrm{~ns}$ after the MD simulations. In addition, the energy contribution of each residue to the protein and ligand interaction was analyzed through the decomposition of the binding free energy. In those methods, the binding free energy ( $\Delta$ Gbind) is deemed as the discrepancy of binding free energies among protein-ligand complex (Gcomplex), the receptor (Gprotein) and ligand (Gligand). The $\Delta$ Gbind can be separated into the electrostatic (Eele) and van der Waals (EvdW) terms, that account for the interaction of the ligand with the receptor in gas-phase, the electrostatic/polar solvation free energy (GGB) and a nonpolar solvation free energy (GSA) refer to the interaction with the solvent, and -T $\Delta \mathrm{S}$ that accounts for the variation of the conformational entropy, which was evaluated from the 1000 frames randomly collected from the equilibrium trajectories using the nmode program in AMBER 16.0. ${ }^{17}$ 
Multiple sequence alignment of the catalytic domains of eight HS2STs. All Hs2STs within Uniprot $\mathrm{KB}$ (https://www.uniprot.org/uniprot/) with a heuristic score $>4$ and reviewed by UniProtKB curators were used in the alignment. Alignment was saved as ALN filetype and submitted to ESPrip ${ }^{18}$ for functional annotation.
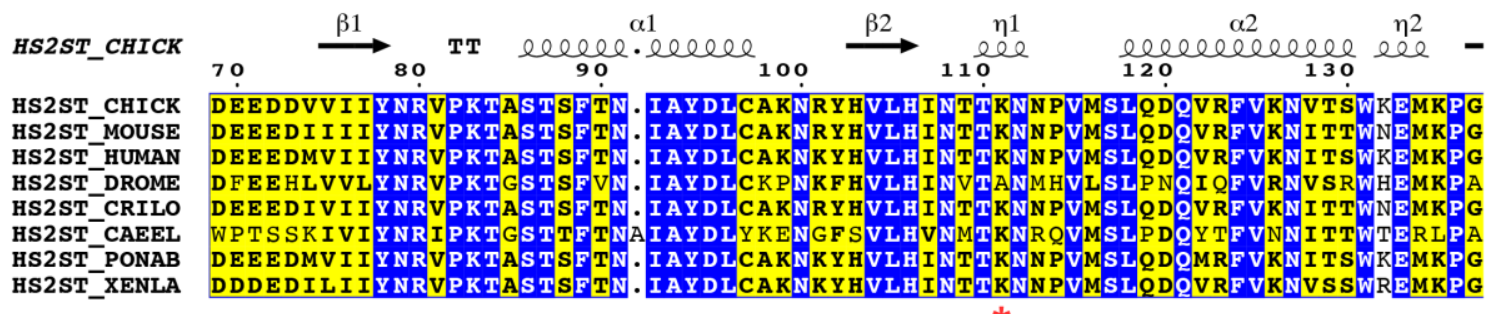
DDDEDILI IYNRVPKTASTSFTN. I AY DLCAKNKY HVLHINTTKNN PVMSLQDQVRF VKNVSSWREMKPG

$\begin{array}{ll}\text { HS2ST_CHICK } & \\ \text { HS2ST_CHICK } & \text { F } \\ \text { HS2ST_MOUSE } & \text { F } \\ \text { HS2ST_HUMAN } & \text { F } \\ \text { HS2ST_DROME } & \text { I } \\ \text { HS2ST_CRILO } & \text { F } \\ \text { HS2ST_CAEEL } & \text { F } \\ \text { HS2ST_PONAB } & \text { F } \\ \text { HS2ST_XENLA } & \text { F } \\ \end{array}$

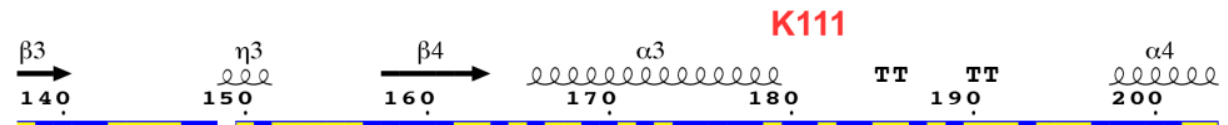

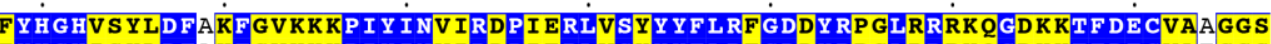
FYH GHI SYLDFAKF GVKKKPIY INVIRDPI ERLVSYYYFLRFGDDYRPGLRRRKOGDKKTF DECVAEGGS FYHGHVSYLDFAKFGVKKKPIY INVIRDPI ERLVSYYYF LRFGDDYRPGLRRRKOGDKKTFDECVAEGGS Y H GHMA F L D F S K F I A K K I Y I NLVRKPL DRLVSYY Y F LRF GDNYRP NLVRKKA GNK ITF DECVVQKQP FYHGHIS Y LDFAKFGVKKKP I Y INVIRDPI ERLVSYYYF LRF GDDY RPGLRRRKQGDKKTFDECVAEGGS FYH GHVAF I DFQRF I A. NP I Y INI IREPLERLLSHY Y F LRY G DNYRI GLKRSRA GNNETF DECYSRGGK Y H GHVS YLDFAKF GVKKKP I Y I NV IRDPI ERLVSYY Y LRF GDDY RP GLRRRKQGDKKTF DECVAEGGS FYHGHV SFLDFTKFIVKKKPI INV IRDPI RLVSYYY FLRFGDDYRPGLRRRKOGDKKTFDECVAAGG

R*

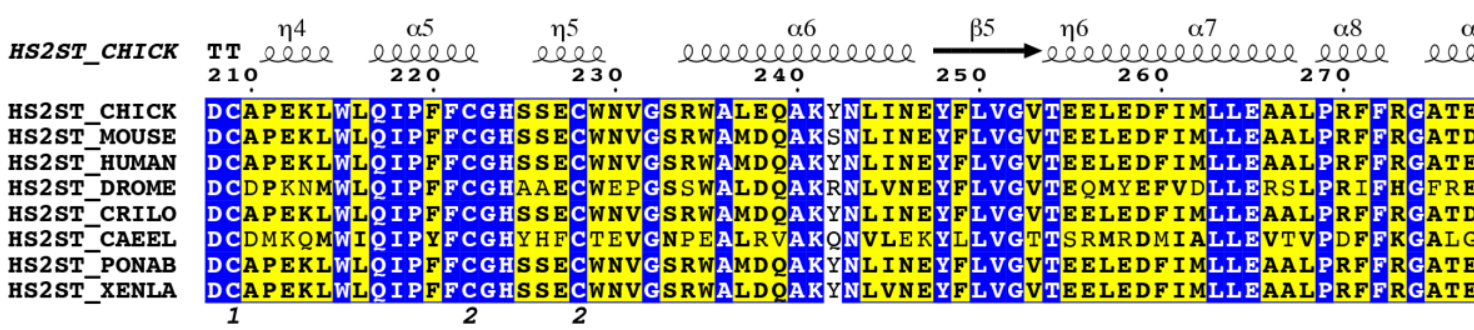

HS2ST_CHICK
280
290

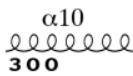

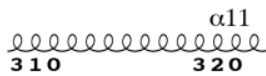
$\underset{330}{\beta 6} \rightarrow \mathrm{TT} \underset{340}{\stackrel{\beta 7}{\longrightarrow}}$

HS2ST_CHICK HS2ST_MOUSE HS2ST_HUMAN HS2ST_DROME HS2ST_CRILO HS2STCAEET HS2ST_PONAB HS2ST_XENLA

LYRT . . GKK SHLRKTTEKK LPTKETIAKLQQSE I WKMENE FYEFALE QFQFVRAHAV . . REKDGELY ILA LYRT . . GKKSHLRKTTEKKLPTKOTIAKLQQSDIWKMENEFYEFALEQFQF IRAHAV . REKDGDLYILA LYRT . . GKKSHLRKTTEKKLPTKQTIAKLQQSDIWKMENEFYEFALEQFQF IRAHAV. . REKDGDLYILA HYHN . . SNKSHLRVTS SKL PPSESTIKS IQKTK IWOMENDLYDFALAOFEFNKKKLM . . Q PDNK . . . HV LYRT. . GKK SHLRKTTEKKLPTKOTIAKLOOSDIWKMENE FYEFALEOFOF IRAHAV, REKDGDLY ILA HFD S LDANRAHLRYTKKK I PPNDOTLSMIRRDEVYKMERE FYDFINNLF DAVFKKATNG I SKADDLVKLP LYRT . . GKKSHLRKTTEKKLPTKOTIAKLQQSD I WKMENE FYEFALEQFQF I RAHAV . . REKD GDLY ILA LYRS. . GKKSHLRKTTEKKAPSKETTAKLQQSDIWKMENEFYEFALEQFQFVRAHAV . REKDGELYVLA * R288

\begin{tabular}{|c|c|}
\hline $2 S T$ CHI & 350 \\
\hline HS2ST_CHIC & QNFFYEKIYPKSN \\
\hline HS2ST_MOUSE & QNFFYEKI \\
\hline HS2ST_HUMAN & QNFFYEKIYPKS N \\
\hline HS2ST_DROME & QKFMYEKIRPK. \\
\hline HS2ST_CRILO & QNFFYEKIYPKSN \\
\hline HS2ST_CAEEL & LQY HFEKIKPS. \\
\hline HS2ST_PONAB & QNFFYEKIYPKSN \\
\hline HS2ST_XENLA & PNFFYEKIYPKSN \\
\hline & K350 \\
\hline
\end{tabular}

Figure S1. Multiple sequence alignment of HS2ST from different species. Regions of HS2ST containing the K111/R188 and R288/K350 talons are evidenced in red and purple, respectively, the ARG triad is evidenced with a green asterisk. Sequence similarities and secondary structure information rendered using ESPript - http://espript.ibcp. fr ${ }^{18}$. The input files used were G. gallus, Q76KB1; M. musculus, Q8R3H7; H. sapiens, Q7LGA3; D. melanogaster, P25722; C. longicaudatus, O08889; C. elegans, O17645; P. abelii, Q5R621; X. laevis, O93336. 
Enzyme assays. The catalytic domain of chicken HS2ST (D69-N356) was cloned and expressed in fusion with the maltose binding protein as described elsewhere ${ }^{19}$. In brief, the MBP-2OST fusion protein was expressed in BL21(DE3) competent cells (New England Biolabs). Cells were grown on a shaker at $37{ }^{\circ} \mathrm{C}$ in LB medium and induced with isopropyl- $\beta$-Dthiogalactopyranoside. Cells were allowed to shake overnight at $16^{\circ} \mathrm{C}$ then pelleted, resuspended in $50 \mathrm{~mm} \mathrm{MES} \mathrm{pH} 7.0,5$ $\mathrm{mM} \mathrm{MgCl}, 10 \mathrm{~mm} \mathrm{MnCl}, 1 \%$ Triton X-100 and cOmplete ${ }^{\mathrm{TM}}$ Protease Inhibitor Cocktail (Roche) and then lysed by sonication. MBP-2OST was bound to amylose resin (New England Biolabs), eluted with 40mM maltose and buffer and buffer exchanged with lysis buffer with $5 \mathrm{mM}$ Maltose. The expression level was monitored by a $10 \%$ Tris$\mathrm{HCl} /$ polyacrylamide gel (Bio-Rad), and the gel was then stained by Coomassie blue. The expression level for each of the analyzed mutants was comparable with the WT protein. Sulfotransferase activity was determined by incubating $5 \mu \mathrm{g}$ of purified WT or mutant HS2ST proteins with $100 \mu \mathrm{g} \mathrm{N}$-sulfo heparosan prepared as previously described ${ }^{20}$. The reaction was incubated at $37{ }^{\circ} \mathrm{C}$ for $120 \mathrm{~min}$ and quenched by the addition of a buffer containing $50 \mathrm{mM} \mathrm{NaOAc}(\mathrm{pH} 5.0)$. Building block analysis were performed in a by Strong-Anion-Exchange HPLC using a 3000 Ultimate Dionex system with a $4.6 x 250$ $\mathrm{mm}$ Waters Spherisorb analytical column $(5 \mu \mathrm{m}$ particle size $)$ at $25^{\circ} \mathrm{C}$ after exhaustive enzymatic digestion with a mixture of heparitinase I, II and III, as previously described ${ }^{21}$. Heparin Disaccharide Standard Mix used as a standard (HD Mix, iduron - https://iduron.co.uk/product/unsaturated-heparin-disaccharides) with the following disaccharide standards eluting: $\Delta \mathrm{UA}-\mathrm{GlcNAc}$ at $4.5 \mathrm{~min}, \Delta \mathrm{UA}-\mathrm{GlcNS}$ at $14.2 \mathrm{~min}, \Delta \mathrm{UA}-\mathrm{GlcNAc}, 6 \mathrm{~S}$ at $15.2 \mathrm{~min}, \Delta \mathrm{UA}, 2 \mathrm{~S}-\mathrm{GlcNAc}$ at $16 \mathrm{~min}, \Delta \mathrm{UA}$ - GlcNS,6S at $18.2 \mathrm{~min}, \Delta \mathrm{UA}, 2 \mathrm{~S}-$ GlcNS at $18.7 \mathrm{~min}, \Delta \mathrm{UA}, 2 \mathrm{~S}-\mathrm{GlcNAc}, 6 \mathrm{~S}$ at $20 \mathrm{~min}$ and $\Delta \mathrm{UA} 2 \mathrm{~S}-\mathrm{GlcNS}, 6 \mathrm{~S}$ at $21.7 \mathrm{~min}$. The catalytic domain of zebrafish HS6OST3 (K75-S395) was expressed and purified as a fusion with the maltose binding protein as previously described ${ }^{22}$. Sulfotransferase activity was determined by incubating $5 \mu \mathrm{g}$ of purified WT or mutant HS6OST proteins with $100 \mu \mathrm{g}$ 6-O-Desulphated Heparin Oligo dp12 from iduron (https://iduron.co.uk/product/unsaturatedheparin-disaccharides).

\section{Metadynamic simulations.}

Sugar puckering. To study the puckering free energy profile of the difference HS2STs substrates, we applied the welltempered metadynamics approach carried out using the open-source, community-developed PLUMED library. ${ }^{23-25}$ The metadynamics algorithm assumes that the system can be described by a set of collective variables (CVs) that are then subjected the addition of a positive Gaussian potential. ${ }^{26}$ As these Gaussians sum up, the final free energy landscape can then be recovered as the opposite of the sum of all Gaussians. Ring conformations can be described by Cremer and Pople dimensionality reduction for the description of a ring conformation. Likewise, the conformation of the glucuronic sixmembered ring can be described by the three coordinates qx, qy and qz, defined as: ${ }^{27}$

$$
\begin{gathered}
q_{x}=\sqrt{\frac{1}{3}} \sum_{j=1}^{6} z_{j} \cos \left[\frac{2 \pi}{3}(j-1)\right] \\
q_{y}=\sqrt{\frac{1}{3}} \sum_{j=1}^{6} z_{j} \sin \left[\frac{2 \pi}{3}(j-1)\right] \\
q_{z}=\sqrt{\frac{1}{6}} \sum_{j=1}^{6}(-1)^{\left(j-1_{-z j}\right.}
\end{gathered}
$$

where $\mathrm{zj}$ is the perpendicular distance of each atom from the ring- average plane. For plumed, the order of atoms for this torsion is $\mathrm{O} 5-\mathrm{C} 1-\mathrm{C} 2-\mathrm{C} 3-\mathrm{C} 4-\mathrm{C} 5$. The alternative puckers biased at those simulation is only considered as a possible structural factor for distinguishing among reactivity patterns. Each pucker at Cartesian coordinates (pseudorotation dihedrals qx, qy and qz) was represented as a collective variable, allowing each GlcA or IdoA to visit all possible configurational spaces while an additional distance collective variable was added between PAPS Sulfur and uronic reactive O2, to preserve a reactive distance to the active site while allowing active site exploration. For both GlcA and IdoA well-tempered metadynamics simulations, the height/width of the Gaussian terms was set at $1.2 \mathrm{kcal} \cdot \mathrm{mol}-1 / 0.20 \AA$ for CV1, CV2 and CV3 (qx, qy and qz) and $1.2 \mathrm{kcal} / \mathrm{mol} / 0.05 \AA$ (CV4, distance) and a new Gaussian-like potential was added every $500 \mathrm{MD}$ steps. Walls for each CV at appropriate distances were used to reduce the Free Energy Landscape (FEL) space to the pucker event. The addition of CV4 (distance from active site) increases total CV dimensionality therefore the addition of parallel tempering is virtually unfeasible. All energies reported in the following are referred to the global minimum. The convergence of the results was checked by calculating Free Energy Profiles corresponding to different simulation times by using the sum_hills tool of PLUMED. ${ }^{24}$ The calculated Free Energy Profiles are three dimensional and can be used for recovering several low dimensional projections of the free energy along the chosen coordinate(s). All data and PLUMED input files required to reproduce the results reported in this paper are available on PLUMEDNEST (www.plumed-nest.org), the public repository of the PLUMED consortium, ${ }^{23}$ as plumID:21001. 
Water coordination. After equilibration, the influence of water shell into the binding free energy of the HS6ST substrate was computed using parallel tempering well-tempered metadynamics in the well-tempered ensemble, ${ }^{25,26}$ using 12 replicas covering the 300- to 360-K temperature range. As presented in Results and Discussion, a set of two CVs was used: CVdist is the distance between the substrate N-sulfoglucosamine and the PAPS sulfate and the coordination is the number of waters surrounding the catalytic H153 and the accessory W158. Hills were deposited every 500 integration steps, with an initial height of $1.5 \mathrm{~kJ} / \mathrm{mol}$ and a bias factor of 15 . The Gaussian sigma was set to $0.05 \mathrm{~nm}$ for all CVs. The metadynamics simulations were terminated when thorough exploration of the relevant CV space was achieved, and the estimates of activation free energy adopted an asymptotic behavior. Metadynamics production runs were performed with a total of 1.7 $\mu$ s of sampling.

QM/MM setup and ONIOM Calculations. The QM/MM ONIOM ${ }^{28,29}$ method, as implemented in Gaussian $16^{30}$ was employed to investigate the reaction mechanisms of the sulfo-group transfer catalyzed by HS2ST and HS6ST enzymes. The system was divided into two regions, treating the high-level (HL) reacting system by density functional theory (DFT) while the low-level (LL) by MM theory. Link atoms ${ }^{31}$ were added to saturate dandling bonds between the layers and the mechanical embedding scheme was used to treat the interaction between the regions. From each MD simulation (HS2ST:GlcA, HS2ST:IdoA and HS6ST:GlcA +4$)$, we monitored i) the distance between the nucleophile $(-\mathrm{OH})$ and electrophile $\left(\mathrm{SO}_{3}{ }^{-}\right)$and ii) the angle between the 5 '-phosphosulfate group and the nucleophile $(\mathrm{O}-\mathrm{S}-\mathrm{O})$. For $\mathrm{HS} 2 \mathrm{ST}:{ }^{1} \mathrm{C}_{4}$-IdoA (see Figure S2), we selected three frames corresponding to the combinations of $2.8 \AA / 157^{\circ}, 2.8 \AA / 137^{\circ}$ and $3.5 \AA / 178^{\circ}$ which could represent the NAC (Near Attack Conformation), which is known to be the gate for the catalytic path that requires the lowest energy (Minimum Energy Path - MEP) for the reaction to proceed. QM/MM optimization of these structures converged in the same conformation, referred as the reactant complex. The MD frame corresponding to the NAC structure was then used as starting point for the QM/MM computations.

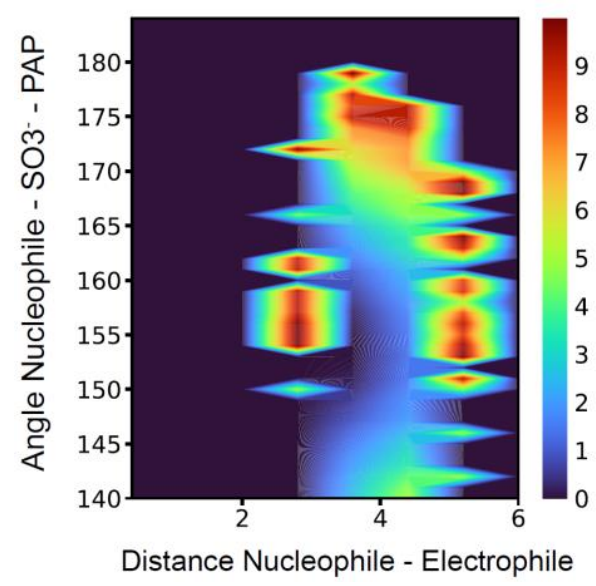

Figure S2. Free energy surface (FES) obtained from HS2ST: ${ }^{1} \mathrm{C}_{4}$-IdoA MD simulation by collecting the distance between the nucleophile $(-\mathrm{OH})$ and electrophile $\left(\mathrm{SO}_{3}{ }^{-}\right)$and the angle between the $5^{\text {' }}$-phosphosulfate group and the nucleophile ( $\mathrm{O}$ $-\mathrm{S}-\mathrm{O})$.

The QM/MM systems under investigation contain all the residues of the monomer A, the sulfo-donor PAPS and the hexasaccharide, for a total of 4949 atoms for HS2ST:GlcA and HS2ST:IdoA and 5385 for HS6ST:GlcA +4 . For HS2ST:GlcA and HS2ST:IdoA systems, the HL comprises the 5'-phosphosulfate group of PAPS, partial sidechains of residues R80, K83, S86, H142, R288 and GlcA (or IdoA respectively) saccharide unit, for a total of 77 atoms treated at the DFT level. The HL model of HS6ST:GlcA +4 systems consist of the 5'-phosphosulfate group of PAPS, the side-chains of residues K104, T108,

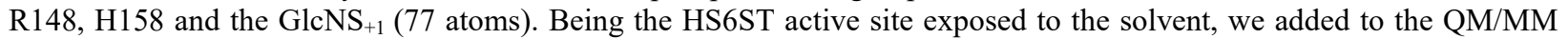
system all the water molecules within $10 \AA$ of the nucleophile atom (O6). To choose the adequate functional to describe the catalysis carried out by the enzymes, we performed single point computations on the $\mathrm{HL}$ of reactant and TS of $\mathrm{HS} 2 \mathrm{ST}:{ }^{4} \mathrm{C}_{1^{-}}$ IdoA system at DLPNO-CCSD(T) level with ORCA (version 4.1.0) software, ${ }^{32}$ using cc-pVTZ and cc-PVTZ/C basis sets. We compared the results obtained ad DLPNO-CCSD(T) with BMK, BMK(+D3), B3LYP, B3LYP(+D3), $\omega$ B97XD, PBEh1PBE and M06-2X functionals. The (+D3) indicates that we explicitly added the Grimme's D3 dispersion correction. ${ }^{33}$ Based on our benchmark results (Table S1) and on previously reported articles, ${ }^{34-38} \mathrm{M} 06-2 \mathrm{X}$ was the functional chosen to describe the HL. Geometry optimizations of the systems were therefore performed at the ONIOM[M06-2X/6$31+\mathrm{G}^{*}:$ ff $14 \mathrm{SB}$ ] level of theory. Linear transit scans along the reaction coordinates were carried out to identify the geometries at the maximum energy, from which transition states (TS) were fully and unrestrictedly optimized. Then, the reactant (Rx) 
and product $(\mathrm{Pd})$ geometries were identified by slightly modifying the geometry in order to fall on one or the other minimum. Frequency calculation resulted in zero imaginary frequencies for minima, and one for transition states, confirming the nature of the points. The activation and reaction free energies reported here were determined as the difference between the Gibbs free energy of TS and Rx, or Pd and Rx, respectively. All the pictures of molecules were created with the VMD, ${ }^{39} \mathrm{Chimera}{ }^{40}$ and Pymol $^{41}$ programs.

Table S1. Activation barriers obtained by DLPNO-CCSD(T)/cc-pVTZ - cc-pvTZ, compared to various functional. All values are given in $\mathrm{kcal} / \mathrm{mol}$.

\begin{tabular}{|c|c|c|}
\hline & $\begin{array}{c}\text { Activation energy } \\
\text { [kcal/mol] }\end{array}$ & Difference with reference \\
\hline $\begin{array}{c}\text { DLPNO-CCSD(T)/cc-pVTZ - cc-pvTZ/C } \\
\text { (reference) }\end{array}$ & 21.6 & 1.7 \\
\hline BMK & 23.4 & 1.1 \\
\hline BMK-D3 & 20.5 & 0.1 \\
\hline wB97-XD & 21.5 & 4.6 \\
\hline B3LYP & 19.0 & 0.2 \\
\hline B3LYP-D3 & 16.7 & 0.1 \\
\hline PBEh1PBE & 21.4 & 21.5 \\
\hline M06-2X & & \\
\hline
\end{tabular}

Ring Pucker analysis. Pyranose ring conformation through the course of MD simulation was determined using Best-fit, Four-Membered Plane, BFMP ${ }^{42}$. To simplify analysis each monosaccharide analyzed was extracted from its trajectory using cpptraj $^{43}$.

Interaction Fingerprinting. Trajectory analysis were performed using a python tool, MD-IFP ${ }^{44}$. In brief, each trajectory was converted to the dcd format using amber's cpptraj tool ${ }^{43}$, the first frame of each MD run converted to pdb format and each monosaccharide composing the GAG polymer also converted to mol2 file format. 200.000 frames of each concatenated molecular dynamics trajectory were used for analysis. Cutoffs for each interaction as defined by MD-IFP.

Grid Inhomogeneous Solvation Analysis. MD simulations were carried on both HS2ST and HS6ST apoproteins to sample water configurations that later could be processed with the GIST approach ${ }^{45-47}$. The system energy was initially minimized using 2500 steps of steepest descent and 2500 steps of conjugate gradient minimization while keeping the non-hydrogen atoms of the solute harmonically restrained to their starting positions with a force constant of $25 \mathrm{kcal} \cdot \mathrm{mol}^{-1} \cdot \AA^{-2}$. A second minimization run was performed with 2500 steps of steepest descent and 2500 steps of conjugate-gradient minimization with a weaker force constant of $25 \mathrm{kcal} \cdot \mathrm{mol}^{-1} \cdot \AA^{-2}$. The system is then heated to $300 \mathrm{~K}$ within $25 \mathrm{ps}$ using an integration time step of $1 \mathrm{fs}$ and positional restraints with a force constant of $25 \mathrm{kcal} \cdot \mathrm{mol}^{-1} \cdot \AA^{-2}$. At a temperature of $300 \mathrm{~K}$, the system was equilibrated to a target pressure of 1 bar using the Berendsen barostat 48 within $5 \mathrm{~ns}$ and at a final equilibration run the system was simulated for $50 \mathrm{~ns}$ in the NVT ensemble. Triplicate production MD runs were carried out for $50 \mathrm{~ns}$ each. 


\section{A}
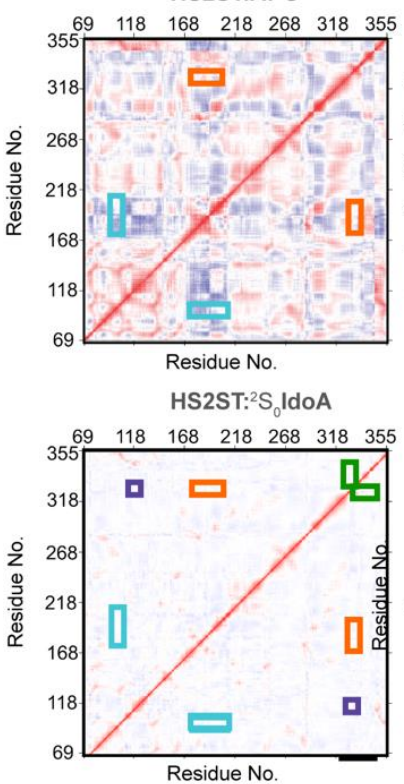

Residue No.

B

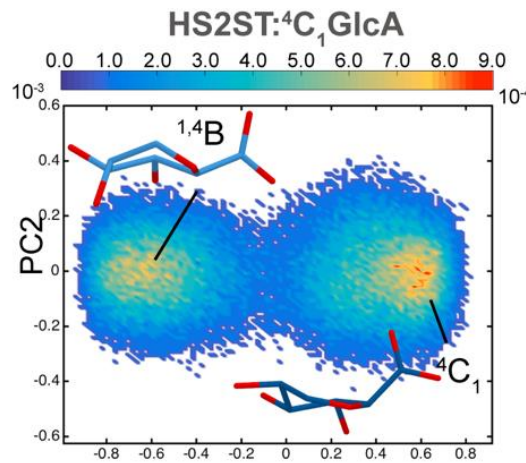

HS2ST: ${ }^{4} \mathrm{C}_{1} \mathrm{GICA}$

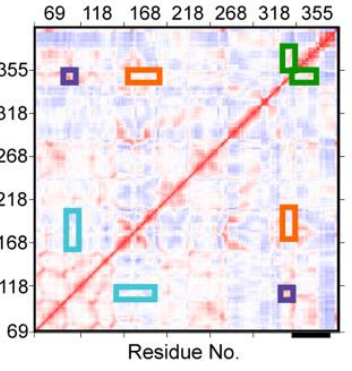

HS2ST: ${ }^{1} \mathrm{C}_{4}$ GIcA

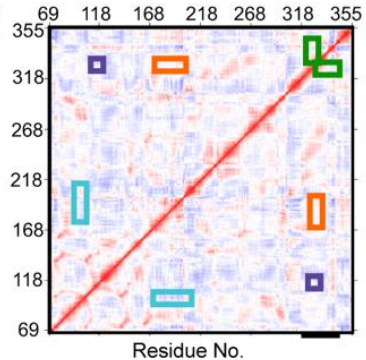

HS2ST: ${ }^{4} \mathrm{C}_{1}$ IdoA

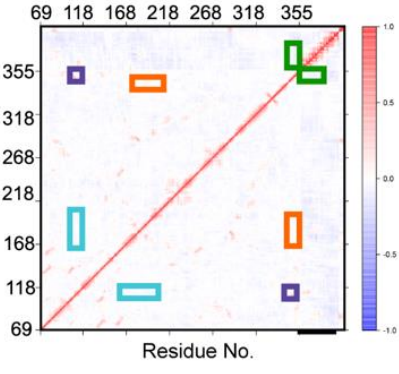

HS2ST: ${ }^{1} \mathrm{C}_{4}$ IdoA

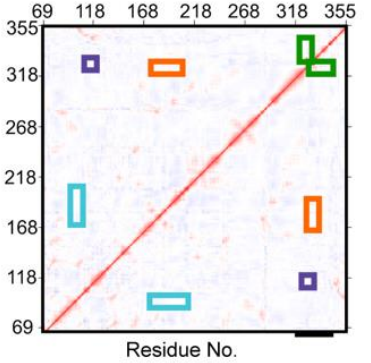

HS2ST: ${ }^{4} C_{1}$ IdoA

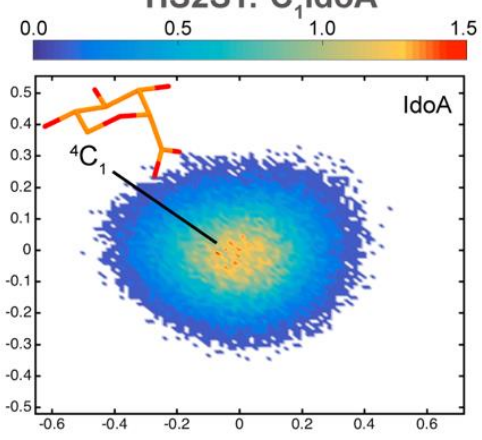

PC1

Figure S3. (A) Cooperative motions underlying the functional dynamics in the apoprotein and sugar bound HS2STs. Perresidue Pearson's coefficients cross correlation matrix derived from the mass-weighted covariance matrix constructed over the last $1.2 \mu \mathrm{s}$ of MD simulations for $\mathrm{C} \alpha$, sugar ring heavy atoms and PAPS nucleoside heavy atoms. CCs are comprised between -1 (anticorrelation, blue) and +1 (correlation, red). The C-terminus tail from the adjacent monomer motions also correlate with the high motility loop (orange rectangles) but is negatively correlated with K111 talon (blue squares). Anticorrelated motions can similarly be observed between glycan with both C-terminus tail from the adjacent monomer and $\mathrm{K} 111$ in the GlcA but not IdoA runs (green rectangles). (B) Dihedral PCA using O5-C1-C2-C3. The diagrams are pseudocolor representations of the density functions $\Delta G=-k_{B} \ln \left(p / p_{\max }\right)$. corresponding to the fluctuations of the O5-C1-C2$\mathrm{C} 3$ atoms on the top three eigenvectors. 


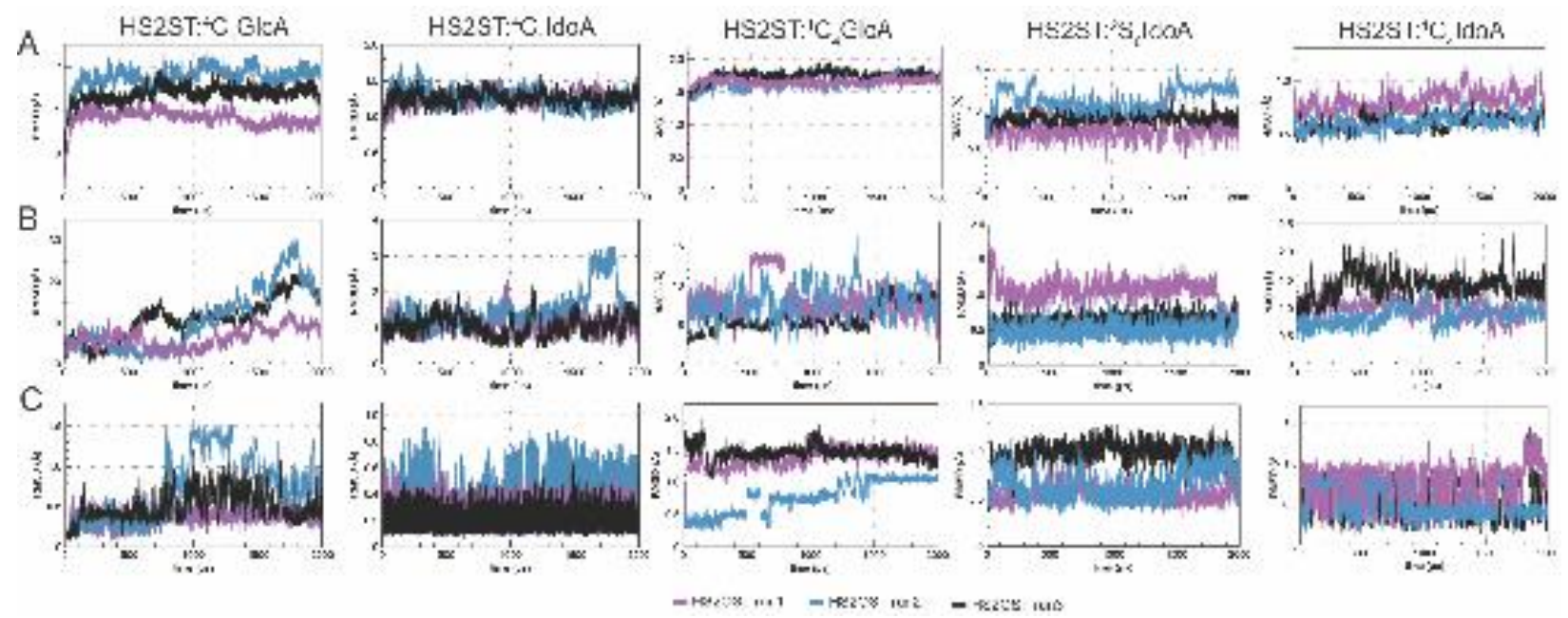

Figure S4. Root mean square deviations conformations (RMSD) for HS2STs (n=3). (A) RMSD of the enzyme alone, (B) RMSD of the enzyme with the oligosaccharide substrate, and (C) RMSD of the enzyme with the oligosaccharide substrate and PAPS, during HS2ST: ${ }^{4} \mathrm{C}_{1}$-IdoA, HS2ST: ${ }^{4} \mathrm{C}_{1}$-GlcA, HS2ST: ${ }^{1} \mathrm{C}_{4}$-IdoA, HS2ST: ${ }^{2} \mathrm{~S}_{0}-\mathrm{IdoA}$ and HS2ST: ${ }^{1} \mathrm{C}_{4}-\mathrm{GlcA}$ simulations.

Discussion S1. In cross correlation plots, red regions represent positive values (DCC score +1$)$ indicating strong correlated motions, while blue regions stand for negative values (DCC score -1) and are associated with anti-correlated movements (Figure S3). ${ }^{48}$ The substrate-free HS2ST $\mathrm{T}_{\text {apo }}$ enzyme displayed the largest correlated fluctuations (Figure S3, left panel), while the HS2ST: ${ }^{4} \mathrm{C}_{1}$-GlcA (Figure S3, central panel), and HS2ST: ${ }^{4} \mathrm{C}_{1}$-IdoA (Figure S3, right panel) showed a decreased flexibility, which was manifested by the decreased cross-correlation matrix map intensity. Indeed, $\mathrm{HS}_{2} \mathrm{ST}{ }^{4} \mathrm{C}_{1}$-IdoA had very subtle correlated motions, recalling the RMSD values that were an average of $0.42 \AA$ lower than the ones found for the $\mathrm{HS} 2 \mathrm{ST}:{ }^{4} \mathrm{C}_{1}$-GlcA simulations (Figure S4), suggesting that the enzyme binds preferably ${ }^{4} \mathrm{C}_{1}$-IdoA. Movement of the high motility loop residues 180-197 of $\mathrm{HS}_{2} \mathrm{ST}_{\text {apo }}$ was negatively correlated with the opposing K111 clamp, adopting several different conformations (cyan rectangles on Figure S3). The adjacent monomer's swapped C-terminus tail (residues 343'355') also points to an intra-correlated motion role towards this region (orange rectangles) while approaching the K111 located helix (purple squares). The hexasaccharide substrate (green rectangles) bound in the $\mathrm{HS} 2 \mathrm{ST}:{ }^{4} \mathrm{C}_{1}$-GlcA and $\mathrm{HS} 2 \mathrm{ST}:{ }^{4} \mathrm{C}_{1}$-IdoA complexes, also showed a decreased flexibility. Finally, the hexasaccharide was negatively correlated with K111 and the swapped C-terminus tail (green rectangles) in the HS2ST:GlcA complex, but not at the HS2ST:IdoA complex, pointing to a higher functional interaction with the later. HS2ST: ${ }^{1} \mathrm{C}_{4}-\mathrm{IdoA}$ and HS2ST: ${ }^{2} \mathrm{~S}_{0}-\mathrm{IdoA}$ correlation plots recall most motions observed at HS2ST: ${ }^{4} \mathrm{C}_{1}$-IdoA, while during the $\mathrm{HS} 2 \mathrm{ST}:{ }^{1} \mathrm{C}_{4}$-GlcA simulations the enzyme shows a marketed anticorrelation at the K111 clamp (cyan squares), indicating a clamp opening. 
A

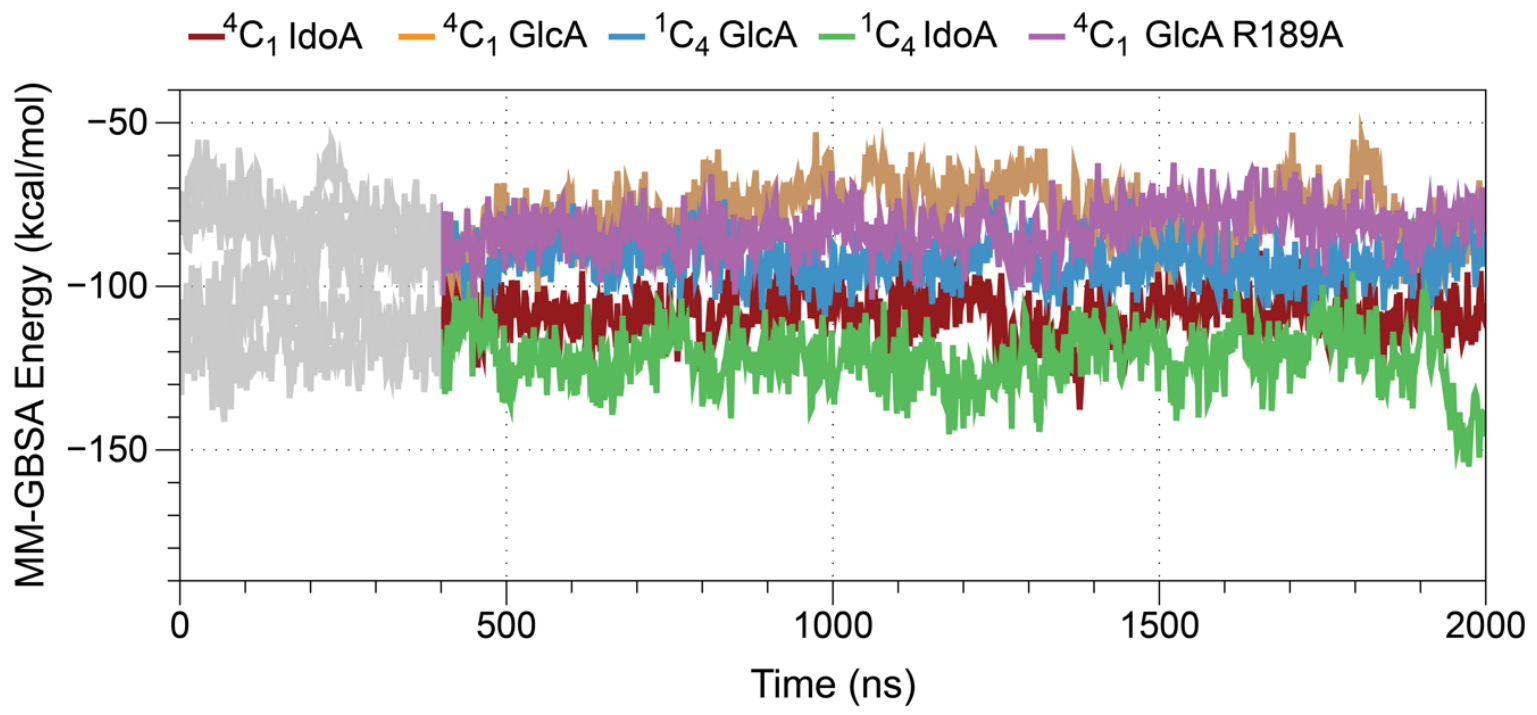

$\mathrm{B}$

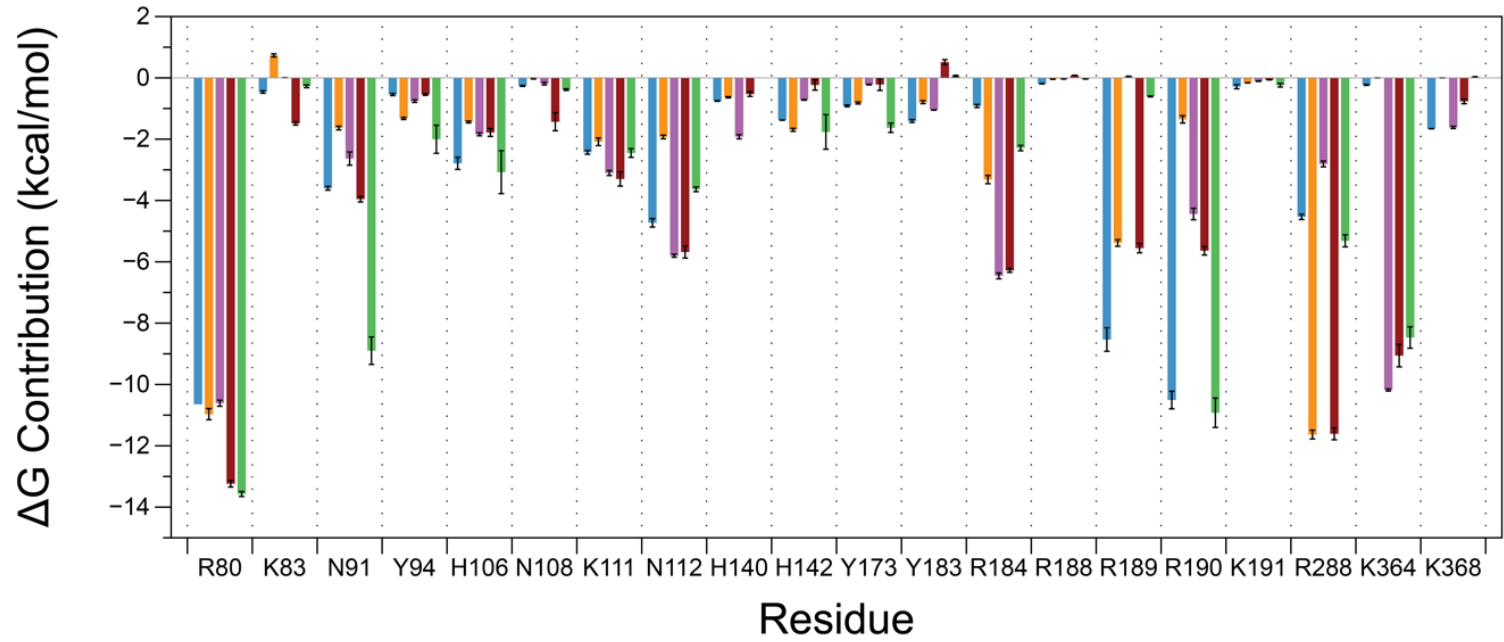

Figure S5. (A) MM-GBSA over time $(2 \mu \mathrm{s})$ as calculated for HS2ST conditions studied and (B) MM-GBSA per-residue contributions to total binding energy of oligosaccharide. Per-residue total free energy contribution of oligosaccharide interfacing amino acids to the stability of ${ }^{4} \mathrm{C}_{1}$-IdoA (vine), ${ }^{4} \mathrm{C}_{1}$-GlcA (orange), ${ }^{1} \mathrm{C}_{4}$-GlcA (blue), ${ }^{1} \mathrm{C}_{4}$-IdoA (green) and mutant ${ }^{4} \mathrm{C}_{1}$-GlcA-189A (purple) calculated with the MM-GBSA method. 
Table S2. MM-GBSA computed binding free energies $(\Delta \mathrm{G})$ for HS2ST complexes formation. The total binding is divided into the electrostatic $\left(\mathrm{E}_{\mathrm{El}}\right)$, van der Waals $\left(\mathrm{E}_{\mathrm{VDW}}\right)$, polar solvation $\left(\mathrm{E}_{\mathrm{GB}}\right)$ and non-polar solvation $\left(\mathrm{E}_{\mathrm{non} \text {-polar }}\right)$ terms. All energies are reported in $\mathrm{kcal} / \mathrm{mol}$.

\begin{tabular}{|c|c|c|c|c|c|}
\hline Complex & $\mathbf{E}_{\text {El }}$ & $\mathbf{E}_{\text {VDW }}$ & $\mathbf{E}_{\mathbf{G B}}$ & $\mathbf{E}_{\text {non-polar }}$ & $\boldsymbol{\Delta}$ \\
\hline HS2ST: ${ }^{4} \mathrm{C}_{1}-\mathrm{IdoA}$ & -1704.8 & -67.2 & 1695.9 & -12.4 & $-88.5 \pm 1.1$ \\
\hline HS2ST: ${ }^{4} \mathrm{C}_{1}$-GlcA & -1270.0 & -67.9 & 1272.3 & -11.3 & $-76.8 \pm 2.5$ \\
\hline HS2ST: ${ }^{4} \mathrm{C}_{1}$-GlcA R189A & -1551.6 & -64.2 & 13254.0 & -10.6 & $-77.5 \pm 3.0$ \\
\hline HS2ST: ${ }^{1} \mathrm{C}_{4}$-IdoA & -1716.3 & -69.5 & 1714.6 & -11.0 & $-114.7 \pm 1.4$ \\
\hline HS2ST: ${ }^{1} \mathrm{C}_{4}$-GlcA & -1247.2 & -65.8 & 1355.1 & -12.0 & $-81.5 \pm 2.9$ \\
\hline
\end{tabular}

Table S3. Per-residue MM-GBSA computed binding free energies $(\Delta \mathrm{G})$ for HS2ST complexes formation.

\begin{tabular}{|c|c|c|c|c|c|c|}
\hline \multirow{4}{*}{ Clamp 1} & Residue $(\Delta \mathbf{G})$ & ${ }^{4} \mathrm{C}_{1}$-IdoA & ${ }^{4} \mathrm{C}_{1}-\mathrm{GlcA}$ & ${ }^{1} \mathrm{C}_{4}$-GlcA & ${ }^{1} \mathrm{C}_{4}$-IdoA & $\begin{array}{c}{ }^{4} \mathrm{C}_{1}-\text { GlcA- } \\
189 \mathrm{~A} \\
\end{array}$ \\
\hline & $\mathbf{R 8 0}$ & $13.1 \pm 0.6$ & $-10.8 \pm 0.7$ & $-10.6 \pm 0.3$ & $-13.4 \pm 0.0$ & $-10.7 \pm 0.6$ \\
\hline & K111 & $-3.8 \pm 0.0$ & $-2.3 \pm 0.0$ & $-2.4 \pm 1.0$ & $-2.5 \pm 0.1$ & $-3.1 \pm 0.2$ \\
\hline & R190 & $-5.7 \pm 0.7$ & $-1.9 \pm 0.3$ & $-10.5 \pm 0.3$ & $-10.9 \pm 0.1$ & $-4.4 \pm 0.6$ \\
\hline \multirow{3}{*}{ Fork } & R184 & $-6.2 \pm 1.3$ & $-3.3 \pm 0.7$ & $-0.9 \pm 0.5$ & $-2.2 \pm 0.2$ & $-6.4 \pm 0.0$ \\
\hline & R189 & $-5.6 \pm 0.1$ & $-5.3 \pm 0.0$ & $-8.5 \pm 0.3$ & $-2.7 \pm 0.8$ & $0.5 \pm 0.7$ \\
\hline & R288 & $-12.1 \pm 0.6$ & $-11.8 \pm 0.7$ & $-4.6 \pm 0.9$ & $-5.3 \pm 0.4$ & $-2.8 \pm 0.4$ \\
\hline \multirow[t]{2}{*}{ Clamp2 } & K350' & $-18.7 \pm 1.5$ & $-5.8 \pm 2.0$ & $-3.3 \pm 0.1$ & $-19.6 \pm 0.0$ & $-0.2 \pm 0.0$ \\
\hline & K354' & $-8.4 \pm 2.1$ & $0.03 \pm 0.0$ & $0.0 \pm 0.0$ & $-8.9 \pm 0.0$ & $-0.0 \pm 0.0$ \\
\hline
\end{tabular}



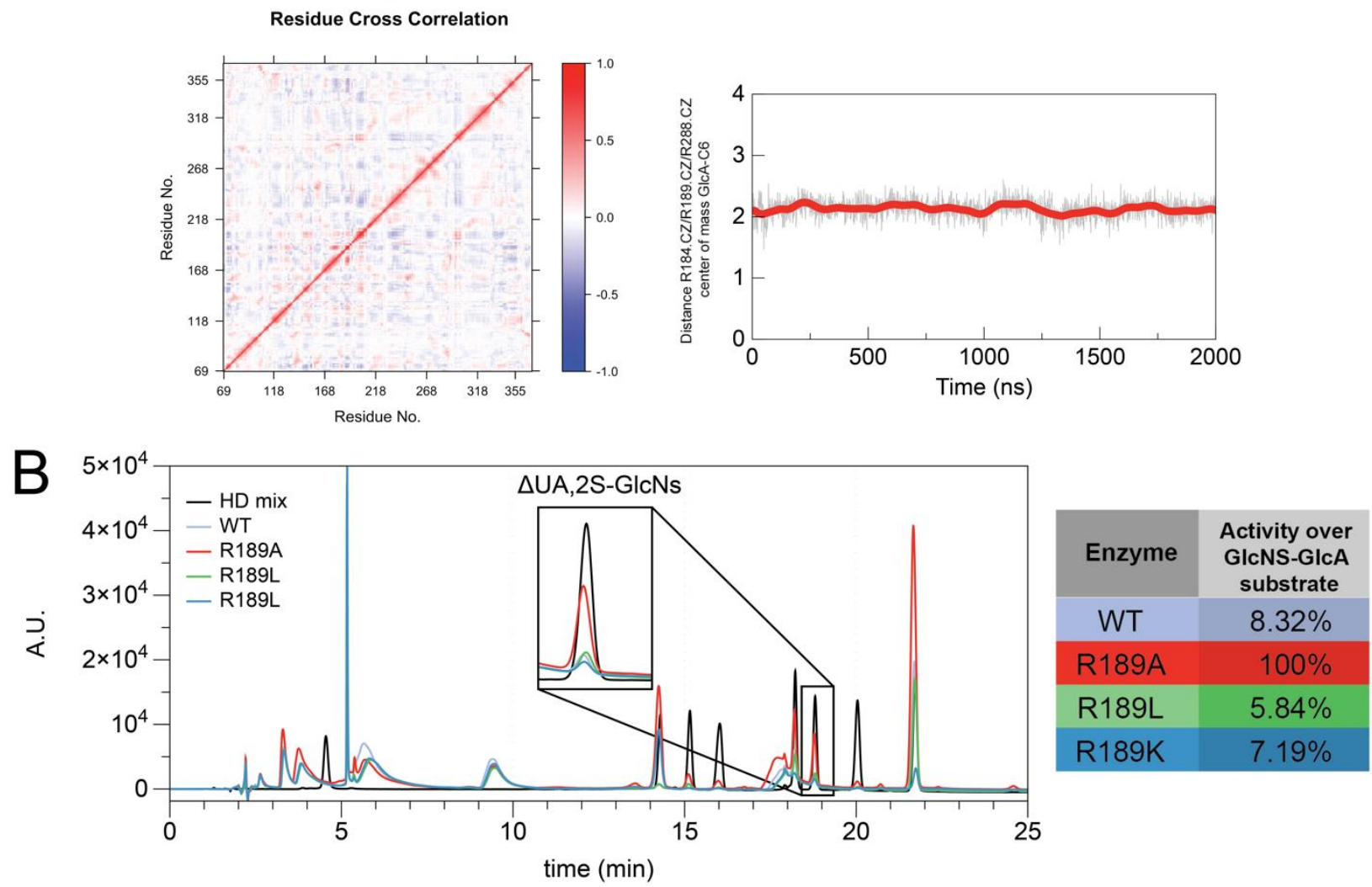

Figure S6. (A) Dynamic-Cross-Correlation of ${ }^{4} \mathrm{C}_{1}$-GlcA R189A simulations. Distance between R184/R189/R288 and hexuronic $\left(\mathrm{C}_{6}\right)$ for HS2ST: ${ }^{4} \mathrm{C}_{1}$-GlcA R189A. (B) SAX-HPLC profile of digested oligosaccharides subjected to wild-type and R189A mutant. Insert highlights the percentage of 2-O-sulfation as measured by the amount of $\Delta \mathrm{UA}, 2 \mathrm{~S}-\mathrm{GlcNS}$ formed by R189A mutant HS2STs in comparison with the wild-type enzyme. A heparin disaccharide standard mix was used as a standard. 

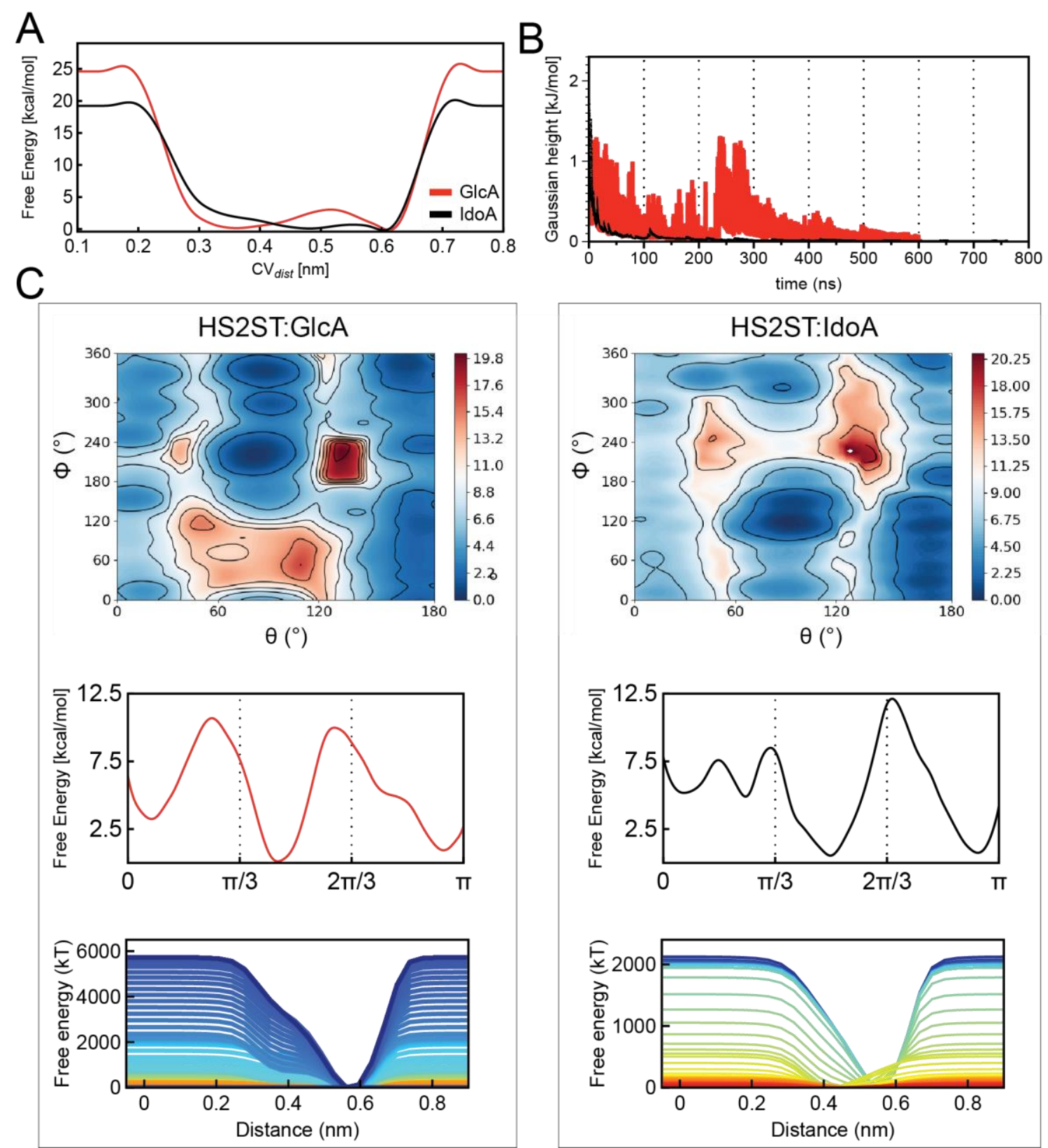

Figure S7. Puckering metadynamics. (A) Free energy landscape as a function of the distance between the active site sugar and PAPS sulfate donor. (B) Time evolution of the Gaussian height during the first 800ns of the well-tempered metadynamics simulations. (C) Top panel: Plate Carré projections of puckering free energy $F_{\text {META }}(\theta$, $\phi)$. Each profile is set to zero at the position of the minimum in the ${ }^{4} \mathrm{C}_{1}$ basin $(\theta<\pi / 3)$ and each isoline is drawn every $10 k_{B} T$. Blue represents lower energy values. Center panel: Projections of the free energy profile onto the $\phi=0$ plane. Lower panel: Evolution of the free energy as a function of $\mathrm{CV}_{\text {dist }}$ calculated every 5000 Gaussian kernels deposited. Colo ramp; blue=0 ns, red $=1000 \mathrm{~ns}$. 

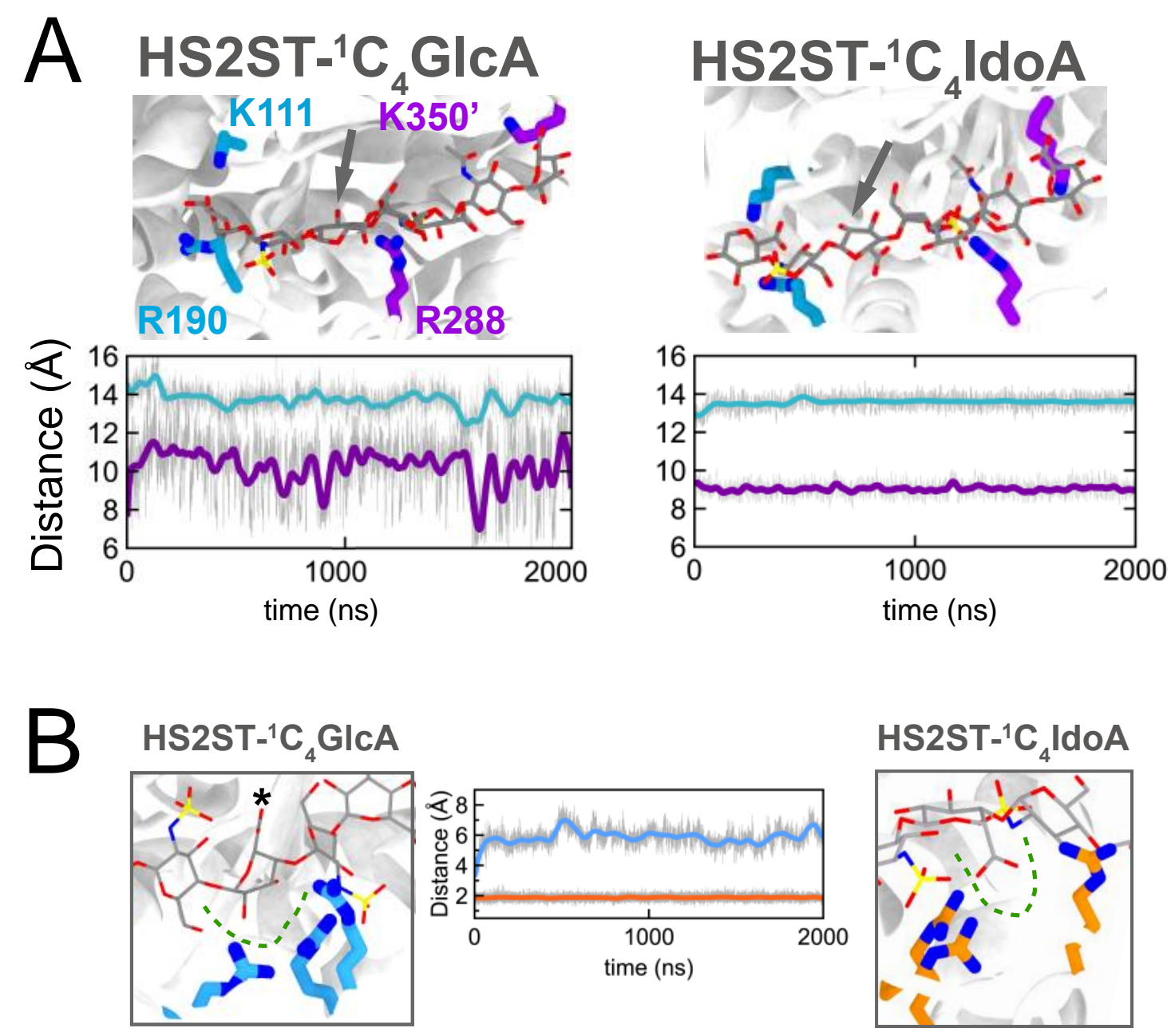

Figure S8. Uronic acid epimers binding to HS2ST. (A) Clamp pairs center of mass distances during simulations with HS2ST: ${ }^{1} \mathrm{C}_{4}-\mathrm{GlcA}$ or HS2ST: ${ }^{1} \mathrm{C}_{4}$-IdoA. (B) Center of mas distance of arginine triad R184/R189/R288 to the C6 atom of either ${ }^{1} \mathrm{C}_{4} \mathrm{IdoA}$ (in orange) or ${ }^{1} \mathrm{C}_{4} \mathrm{GlcA}$ (in blue). 

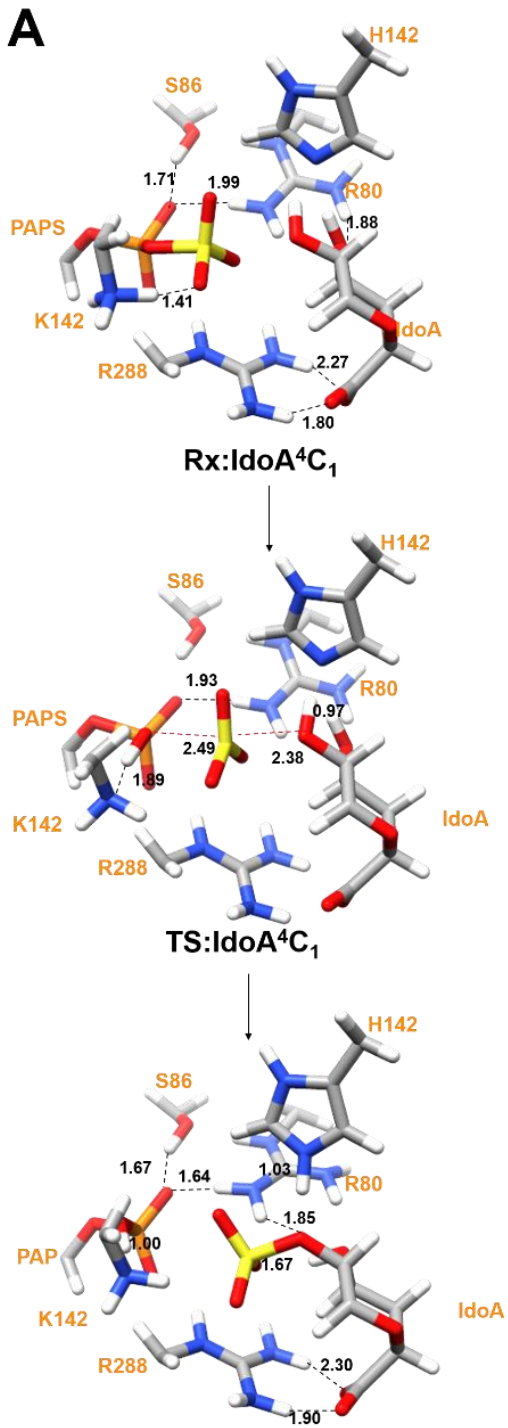

Pd:IdoA ${ }^{4} C_{1}$
B

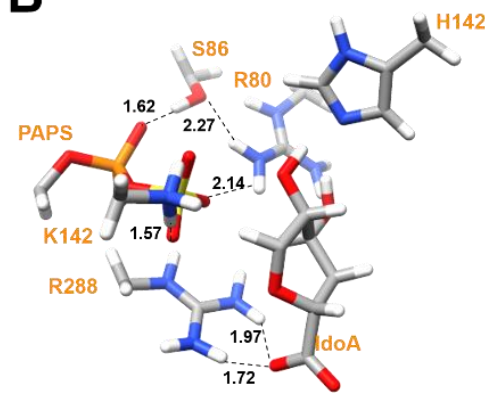

Rx:IdoA ${ }^{2} S_{0}$

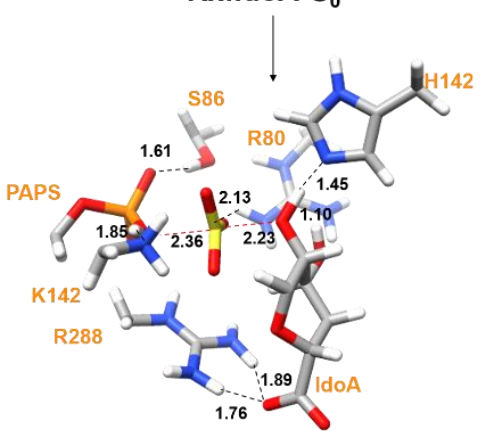

TS:IdoA ${ }^{2} S_{0}$

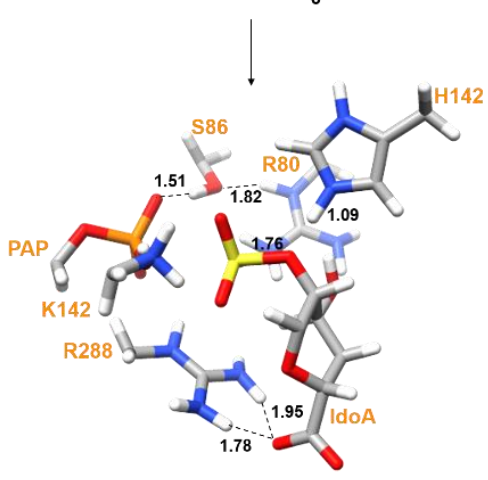

Pd:IdoA $\mathbf{S}_{0}$

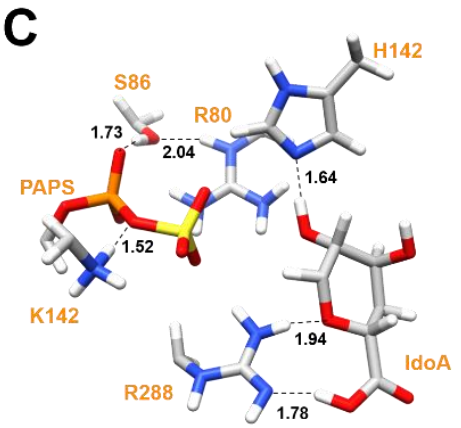

Rx:IdoA ${ }^{1} C_{4}$
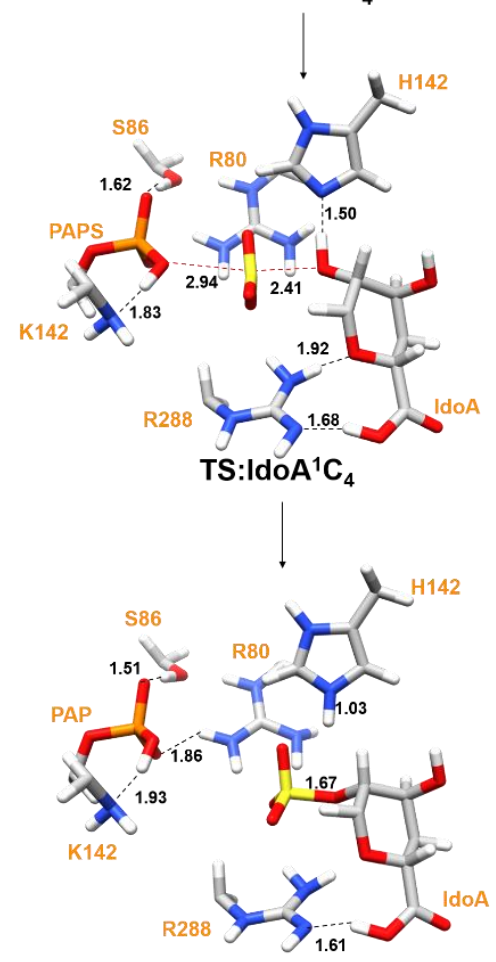

Pd:IdoA ${ }^{1} C_{4}$

Figure S9. 3D-representation of the critical points obtained at the ONIOM[M06-2X/6-31+G*:ff14SB] level of theory for the catalytic mechanism of the sulfate transfer from PAPS to the IdoA acceptor in different conformations: (A) ${ }^{4} \mathrm{C}_{1}$-IdoA, (B) ${ }^{2} \mathrm{~S}_{0}$-IdoA and (C) ${ }^{1} \mathrm{C}_{4}$-IdoA. The distances are reported in Angstroms. 

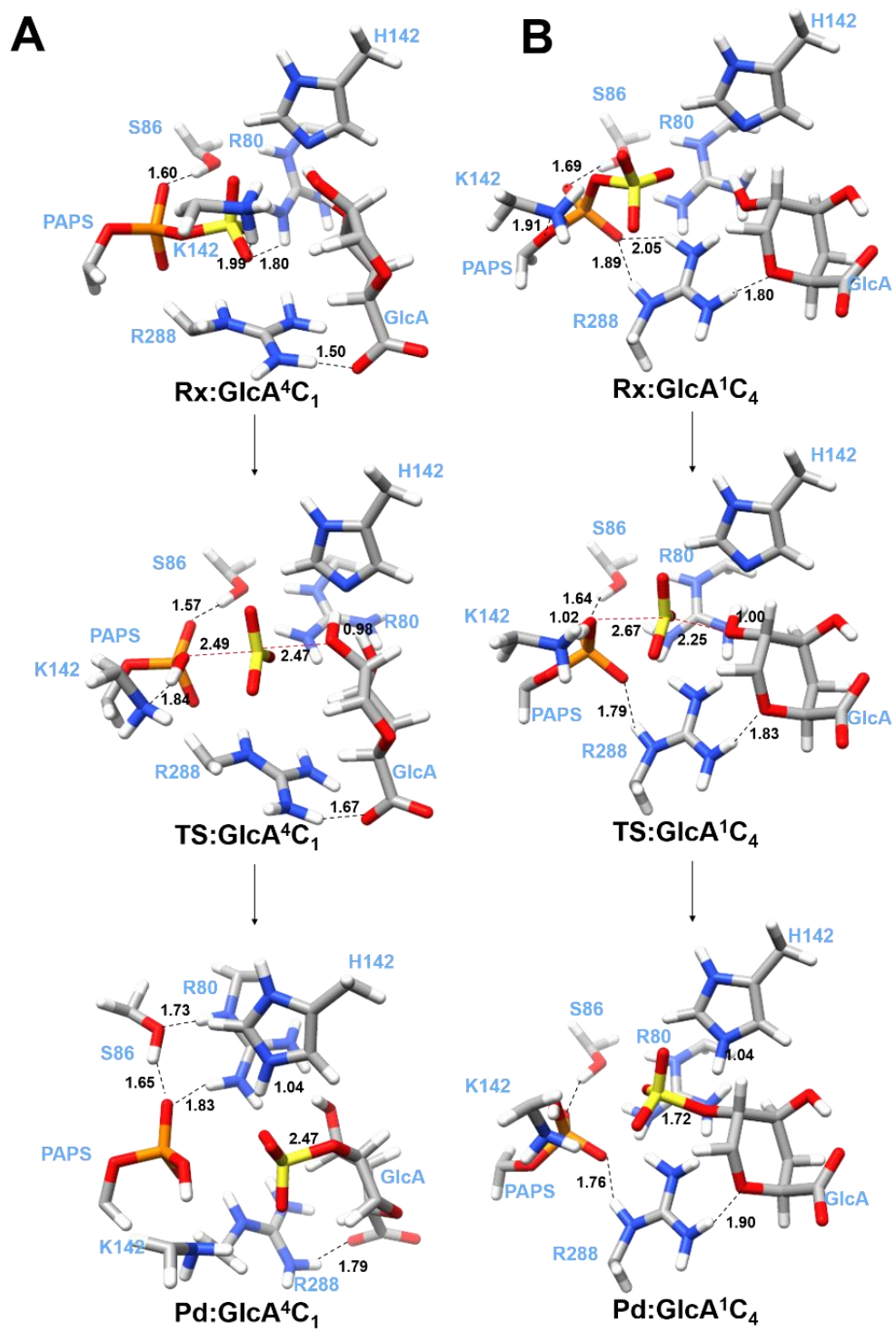

Figure S10. 3D-representation of the critical points obtained at the ONIOM[M06-2X/6-31+G*:ff14SB] level of theory for the catalytic mechanism of the sulfate transfer from PAPS to $(\mathbf{A}){ }^{4} \mathrm{C}_{1}-\mathrm{GlcA}$ and $(\mathbf{B}){ }^{1} \mathrm{C}_{4}-\mathrm{GlcA}$. The distances are reported in Angstroms.

Discussion S2. In order to attain closer insights into the HS2ST catalysis, a detailed description of the catalytic mechanism and the role of the active site residues is herein reported. Also, a 3D-representation of the critical points is shown in Figure S9-S10. In Rx:IdoA ${ }^{4} \mathrm{C}_{1}$ (Figure $\mathrm{S} 9 \mathrm{~A}$, top panel), a network of hydrogen bonds arranges the reactants PAPS and IdoA in a pre-reactive conformation, such that the $O 2$ of IdoA is in line with the O-S bond of the phosphosulfate group of PAPS $\left(172.8^{\circ}\right)$. K83 and S86 interact with the phosphosulfate group of PAPS (1.41 $\AA$ and $1.71 \AA$ respectively), while R288 binds via two hydrogen bonds with the carboxylic group of the iduronic acid $(1.80 \AA$ and $2.27 \AA)$. The other arginine in the catalytic site, R80, anchors both the oxygen of the phosphate $(1.99 \AA)$ and $3 \mathrm{OH}-\mathrm{IdoA}(1.88 \AA)$. The TS:IdoA ${ }^{4} \mathrm{C}_{1}$ (Figure $\mathrm{S} 9_{\mathrm{A}}$, middle panel), describes a $\mathrm{S}_{\mathrm{N}}$ 2-like mechanism, where a planar sulfuryl fragment is migrating from PAPS to IdoA, being placed almost halfway between the two oxygens $(2.49 \AA$ from the donor phosphate and $2.38 \AA$ from the $O 2$ nucleophile). The deprotonation of the nucleophile, acted by H142, takes place later with respect to the $\mathrm{SO}_{3}{ }^{-}$transfer, so that in the TS the $\mathrm{O} 2-\mathrm{H}$ bond is still formed $(0.97 \AA)$. At the transition state, K83 stabilizes the incipient negative charge of the oxygen that is losing the sulfuryl group, by protonating it $(1.89 \AA)$, while R80 is holding both the $\mathrm{PO}_{4}{ }^{2-}(1.93 \AA)$ and the O3$\mathrm{H}(2.11 \AA)$ groups in place. Once the transition state is surmounted, the system collapses into the product complexes. Figure $\mathrm{S} 9 \mathrm{~A}$ (bottom panel) shows $\mathrm{Pd}: \mathrm{IdoA}^{4} \mathrm{C}_{1}$, where the new bond between the oxygen and the sulfur atom can be found (1.67 $\AA$ ) in the new IdoA2S unit and the protonation of histidine is completed (1.03 $\AA$ ). The network of hydrogen bonds is engaged 
by R80 that interacts with $\mathrm{O} 2$ of IdoA2S (1.85 $\AA$ ) and with the phosphate of PAP (1.64 $\AA$ ), and by S86 that stabilizes the phosphate group via a strong hydrogen bond $(1.67 \AA)$, while the guanidine group of R288 anchors the carboxylate group of IdoA2S (hydrogen bonds $1.90 \AA$ and $2.30 \AA$ ).

Considering the ${ }^{2} \mathrm{~S}_{0}$ conformation (Figure $\mathrm{S} 9_{\mathrm{B}}$, top panel), in Rx:IdoA ${ }^{2} \mathrm{~S}_{0}, \mathrm{~K} 83$ and $\mathrm{S} 86$ engage in hydrogen bonding with the phosphosulfate (1.57 $\AA$ and $1.62 \AA$ respectively), R288 anchors the $\mathrm{COO}^{-}$group of ${ }^{2} \mathrm{~S}_{0}$-IdoA $(1.72 \AA)$ and R80 blocks $\mathrm{S} 86(1.73 \AA)$ and the $\mathrm{SO}_{3}{ }^{-}$group of PAPS $(2.14 \AA)$. When the sulfuryl group detaches from PAPS in TS:IdoA ${ }^{2} \mathrm{~S}_{0}(2.36 \AA$ and the $2.23 \AA$ from $2 \mathrm{O}$ ), K83 had completely protonated the phosphate group while the deprotonation of the nucleophile has still to take place $(\mathrm{O}-\mathrm{H} 1.10, \mathrm{H}---\mathrm{N}-\mathrm{H} 1421.45 \AA)$. R288 still interacts with the carboxylated group of IdoA by two strong H-bonds (1.76 and $1.89 \AA$ ), while R80 engages in a weak interaction with the planar $\mathrm{SO}_{3}{ }^{-}$group $(2.13 \AA)$, as represented in Figure $\mathrm{S} 9_{\mathrm{B}}$, middle panel. For Pd:Ido $\mathrm{A}^{2} \mathrm{~S}_{0}$ (Figure $\mathrm{S} 9_{\mathrm{B}}$, bottom panel), a new O2-S bond is formed (1.76 $\AA$ ), the histidine is protonated (N-H 1.09 $\AA$ ) and PAP is protonated on the phosphate group. R80, S86 and R288 engage in a network of hydrogen bonds comparable to that in Rx:IdoA ${ }^{2} \mathrm{~S}_{0}$ and TS:IdoA ${ }^{2} \mathrm{~S}_{0}$.

The reactant complex Rx:Ido $A^{1} \mathrm{C}_{4}$ presents a hydrogen bond between $\mathrm{R} 80$ and $\mathrm{S} 86$ (1.79 $\AA$ ), that, in turn, interacts with 5 '-phosphate moiety of PAPS (1.73 $\AA$ ). While K83 engages an electrostatic interaction with the oxygen of 5'-phosphosulfate $(1.51 \AA), \mathrm{R} 288$ anchors the iduronic acid by H-bond with the endocyclic oxygen $(1.93 \AA)$. Interestingly, the interaction between the guanidinium group of R288 and the carboxylated moiety of IdoA causes the protonation of the negative group. $\mathrm{H} 152$ engages an interaction with the $\mathrm{C} 2-\mathrm{OH}$, holding the substrate in the pre-reactive conformation. The sulfation takes place at TS:IdoA ${ }^{1} \mathrm{C}_{4}$ where the $\mathrm{SO}_{3}{ }^{-}$group is migrating from the donor $(2.94 \AA)$ to the $2-\mathrm{OH}$ acceptor $(2.41 \AA)$; at the transition state $\mathrm{K} 83$ has protonated the incipient negative charge of the phosphate while the nucleophile activation has not taken place yet ( $\left.\mathrm{N}^{\cdots} \mathrm{HO}-\mathrm{C} 21.50 \AA\right)$. R80, S86 and R288 are involved in a hydrogen bond network very similar to that found in the reactant complex. At Pd:IdoA ${ }^{1} \mathrm{C}_{4}$ the formation of the IdoA2S is completed (2O-P $\left.1.67 \AA\right)$ and the catalytic base is protonated $\left(\mathrm{N}-\mathrm{H} 1.03 \AA\right.$ ). While R80 engages $\mathrm{H}$-bond with the $5^{\prime}$ '-phosphate group of PAP, R80 anchors the 2-OSO ${ }_{3}^{-}$group $(1.98 \AA)$. R288 maintains the IdoA2S unit in place by electrostatic interaction with the COOH moiety (1.60). The decreased distance of this last interaction suggests that the ligand, before dissociating from the active site, has to transfer the proton back to R288.

Concerning the results obtained for the sulfation of the 4C1-GlcA by HS2ST and reported in (Figure S10 $\mathrm{A}$, top panel), for reactant complex Rx:GlcA ${ }^{4} \mathrm{C}_{1}, \mathrm{R} 80$ interacts with the phosphosulfate of PAPS by a hydrogen bond (1.89 $\left.\AA\right)$; the charged sidechain of $\mathrm{K} 83$ engages a hydrogen bond with the $\mathrm{SO}_{3}{ }^{-}$moiety $(1.99 \AA)$, S86 is responsible for interacting with an oxygen of the phosphate $(1.60 \AA)$ and R288 anchors the carboxylate of GlcA $(1.50 \AA)$ by a H-bond. At TS: GlcA ${ }^{4} \mathrm{C}_{1}$, the transfer of the sulfuryl group from PAPS to GlcA takes place; while the trigonal planar $\mathrm{SO}_{3}{ }^{-}$migrates from the donor $(2.49 \AA)$ to the acceptor $(2.47 \AA)$, the $O 2$ deprotonation by the catalytic base $\mathrm{H} 142$ has not yet taken place (Figure $\mathrm{S} 10_{\mathrm{A}}$ ). At the transition state (Figure $\mathrm{S}_{10}$, middle panel), all non-covalent interactions found in reactant complexes are maintained, with the exception of K83 that protonates the incipient negative charge on the oxygen atom of the phosphate group in both transition states, being the $\mathrm{N}-\mathrm{H}$ distance $1.84 \AA$. The products shown in Figure $\mathrm{S} 10_{\mathrm{A}}$, bottom panel, are then formed, and in $\mathrm{Pd}$ : $\mathrm{GlcA}{ }^{4} \mathrm{C}_{1}$ the R80 residue engages in hydrogen bonds via its guanidinium moiety with S86 (1.68 $\AA$ ), O3H of GlcA2S (1.94 $\AA$ ) and the oxygen of the PAP phosphate $(1.80 \AA)$.

For the sulfation of ${ }^{1} \mathrm{C}_{4}$ GlcA (Figure $\mathrm{S} 10_{\mathrm{B}}$, top panel) in the reactant complex $\left(\mathrm{Rx}: \mathrm{GlcA}{ }^{4} \mathrm{C}_{1}\right.$ ), the guanidinium group of $\mathrm{R} 80$ is placed a little further from PAPS in respect to $\mathrm{Rx}: \mathrm{GlcA}^{4} \mathrm{C}_{1}$, and the hydrogen bond engaged with the phosphate group is $1.95 \AA$. K83 interacts with the sulfuryl moiety $\left(1.87 \AA\right.$ in $\left.\mathrm{Rx}: \mathrm{GlcA}^{4} \mathrm{C}_{1}\right), \mathrm{S} 86$ with PAPS phosphate $(1.70 \AA)$ and the guanidinium group of R288 is engaged in an electrostatic interaction with the cyclic oxygen of GlcA (1.82 $\AA$ ), since the carboxylated group of the sugar, due to the puckered conformation, points away from the catalytic site. The TS (Figure $\mathrm{S} 10_{\mathrm{B}}$, middle panel) is defined by the migration of the $\mathrm{SO}_{3}{ }^{-}$group that is placed $2.67 \AA$ from the donor (PAPS) and $2.25 \AA$ from the acceptor. Analogously to the other transition states, the nucleophile deprotonation is a late process $(\mathrm{N}---\mathrm{H} 1.63 \AA$ ) while the proton transfer from K83 to the phosphate of PAPS is completed. As reported in Figure $\mathrm{S} 9_{\mathrm{B}}$ (bottom panel), for Pd:GlcA ${ }^{4} C_{1}, \mathrm{R} 80$ and S86 interact with the phosphate group of PAP (1.61 $\AA$ and $1.85 \AA$, respectively), while R288 anchors the ${ }^{1} \mathrm{C}_{4}$-GlcA2S by interacting with its sulfuryl group $(2.68 \AA)$ and the $\mathrm{O} 1(1.90 \AA)$. 

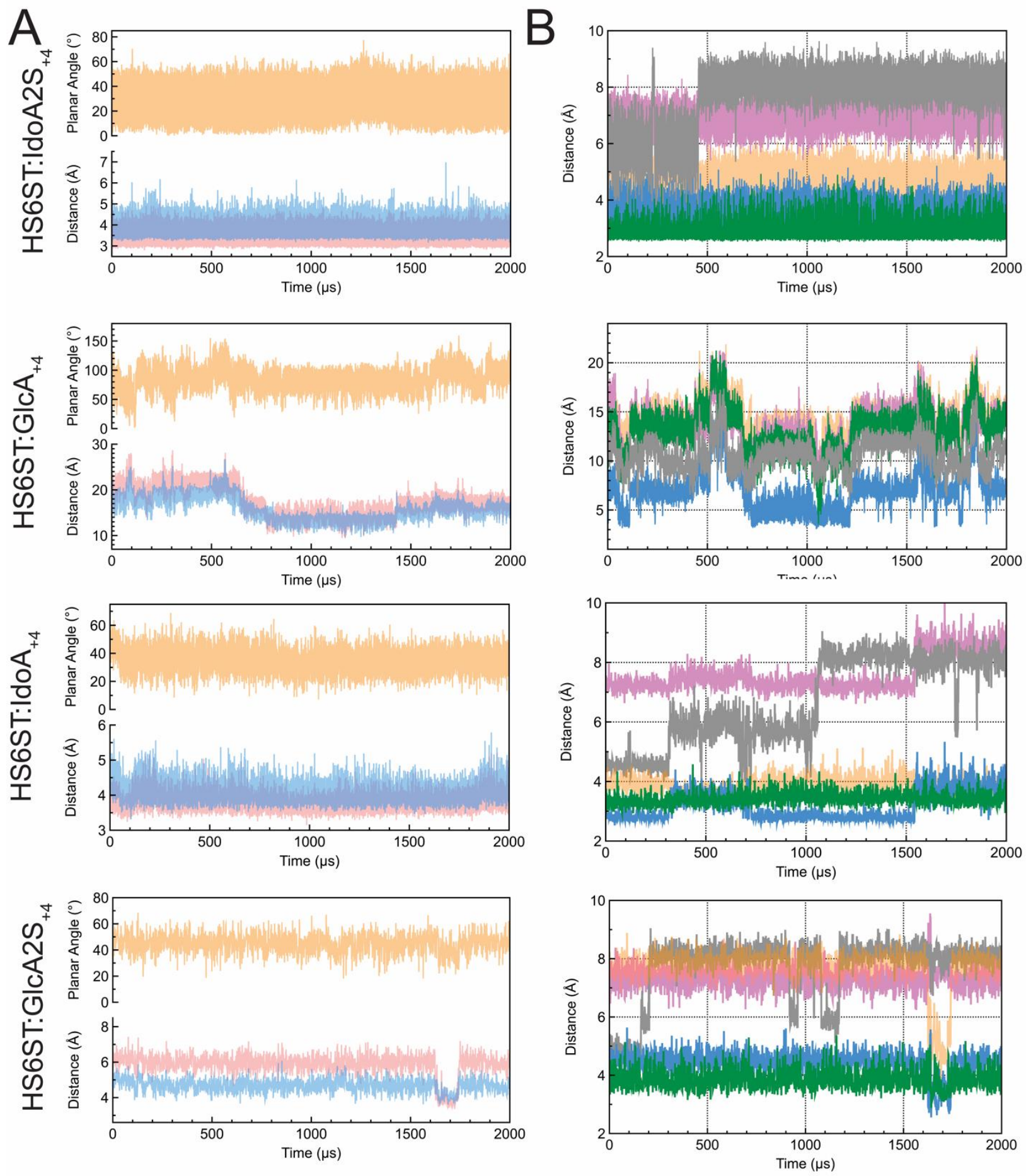

- GlcNS.O6--Q103.CG — GIcNS.O6--H158:NE2 - GlcNS.C4--H203.ND1 — GlcNS.C4--H158.CD2 — GIcNS.C6--R148.NH2

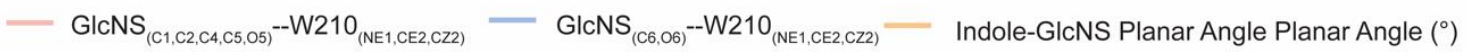

Figure S11. Molecular dynamics analysis of HS6ST. Distance vs planar angle for the GlcNS-W210 pair for HS6ST:IdoA2S ${ }_{+4}$, HS6ST:GlcA +4, HS6ST:IdoA +4 and HS6ST:GlcA2S +4 simulations. (A) Polar angle as calculated between vectors from W210 atoms C1 and C2 indole and acceptor GlcNS CD1 and CZ3 atoms (orange). (B) Distance between critical active site atoms and the substrate -GlcNS with Q103 (yellow), H158 (green/pink), H203 (gray), R148 (blue) also depicted. 
Discussion S3. To verify the existence of a $\mathrm{CH}-\pi$ interaction between $\mathrm{W} 210$ and the acceptor unit GlcNS $\mathrm{S}_{+1}$, two geometrical parameters were chosen: the distance between the $\mathrm{GlcNS}_{+1}$ substrate carbon atom closest to the center of the indole phenyl ring at $\leq 4.5 \AA\left(\mathrm{d}_{\mathrm{CH}-\pi}\right)$ on the one hand and the angle between the $\mathrm{CH}$ vector and the phenyl ring plane normal vector at $\leq 40^{\circ}\left(\omega_{\mathrm{CH}-\pi}\right)$ on the other hand. ${ }^{49-51}$ The distance $\mathrm{d}_{\mathrm{CH}-\pi}$ averages $3.2 \AA$ for HS6ST:IdoA2 $\mathrm{S}_{+4}$ and 15.8 for HS6ST:GlcA +4 , while $\omega_{\mathrm{CH}-\pi}$ averages $29.2^{\circ}$ for HS6ST:IdoA2S ${ }_{+4}$ and $85.4^{\circ}$ for HS6ST:GlcA +4 (Figure S11A and B). This notable difference is due to the displacement of the non-reducing end of the oligosaccharide substrate from the active site of the enzyme in the HS6ST:GlcA+4 complex, as the sugar drifts away from the binding site. The dissociation is not observed in any of the HS6ST:Ido2 $\mathrm{S}_{+4}$ simulations. Simulations with HS6ST:Ido+4 and HS6ST:GlcA2S +4 recapitulate the HS6ST:Ido2 $\mathrm{S}_{+4}$ runs. We also carried out single point QM computations (M06-2X/6-31+G*) on fragment geometries extracted from the HS6ST:IdoA2S ${ }_{+4}$ and HS6ST:GlcA ${ }_{+4}$ (before the drifting of the sugar outside the binding cleft) MD simulations. In all the computations the error caused by the intermolecular superposition of the basis functions of nearby fragments (BSSE) was deleted. ${ }^{49}$ We found that tryptophan W210 interacts strongly with GlcNS +1 in HS6ST:IdoA2S +4 ($31.1 \mathrm{kcal} / \mathrm{mol}$ ), while in HS6ST:GlcA ${ }_{+4}$ the interaction energy accounts for only $-10.0 \mathrm{kcal} / \mathrm{mol}$. This confirms that, when the ligand in inside the binding pocket, HS6ST anchors the IdoA2 $\mathrm{S}_{+4}$ containing ligand stronger than Glc $\mathrm{A}_{+4}$. Also, considering the magnitude of the interaction and comparing it to the available literature, we can confirm that this interaction is not likely to be of a $\mathrm{CH}-\pi$ type, since the reference values reported in literature are about $-0.5 \mathrm{kcal} / \mathrm{mol}$. ${ }^{52}$ 
Table S4. MM-GBSA computed binding free energies $(\Delta \mathrm{G})$ for HS6ST complexes formation. The total binding is divided into the electrostatic $\left(\mathrm{E}_{\mathrm{El}}\right)$, van der Waals $\left(\mathrm{E}_{\mathrm{VDW}}\right)$, polar solvation $\left(\mathrm{E}_{\mathrm{GB}}\right)$ and non-polar solvation $\left(\mathrm{E}_{\text {non-polar }}\right)$ terms. All energies are reported in $\mathrm{kcal} / \mathrm{mol}$.

\begin{tabular}{|c|c|c|c|c|c|}
\hline Complex & $\mathbf{E}_{\text {El }}$ & $\mathbf{E}_{\text {VDW }}$ & $\mathbf{E}_{\mathbf{G B}}$ & $\mathbf{E}_{\text {non-polar }}$ & $\boldsymbol{\Delta}$ \\
\hline HS6ST:GlcA $_{+4}$ & -1133.9 & -26.7 & 1125.6 & -5.2 & $-40.2 \pm 1.6$ \\
\hline HS6ST:IdoA2S $_{+4}$ & -1730.8 & -52.5 & 1633.3 & -9.4 & $-124.2 \pm 1.1$ \\
\hline HS6ST:IdoA & -1138.5 & -59.3 & 1045.6 & -6.8 & $-50.3 \pm 1.4$ \\
\hline HS6ST:GlcA2S & -1412.9 & -31.47 & 1354.7 & -8.7 & $-91.8 \pm 2.0$ \\
\hline
\end{tabular}



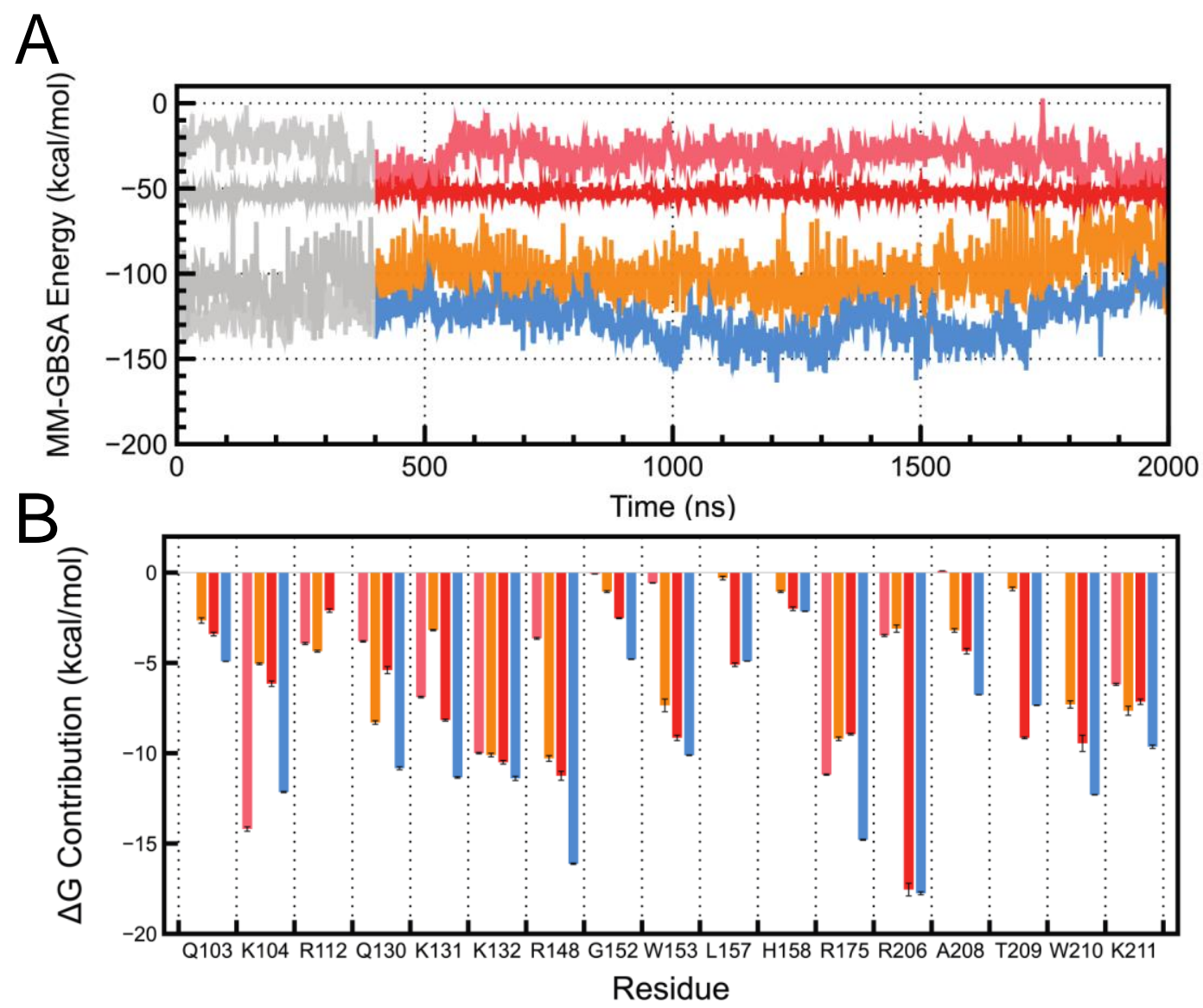

-HS6ST:GlcA ${ }_{+4}-$ HS6ST:IdoA2S $_{+4}-$ HS6ST:GlcA2S $_{+4}-$ HS6ST:IdoA $_{+4}$

Figure S12. (A) MM-GBSA was calculated for HS6ST with IdoA2 $\mathrm{S}_{+4} / \mathrm{GlcA}_{+4} / \mathrm{IdoA}_{+4} / \mathrm{GlcA}_{2} \mathrm{~S}_{+4}$ simulations. MM-GBSA per-residue contributions to total binding energy of oligosaccharide calculated. (B) Per residue total free energy contribution of oligosaccharide interfacing amino acids to the stability of IduA2S $\mathrm{S}_{+4}$ (blue), GlcA + $_{+4}$ (salmon), IduA+4 (red) and GlcA2S $\mathrm{S}_{+4}$ (orange) was calculated with the MM-GBSA method. 


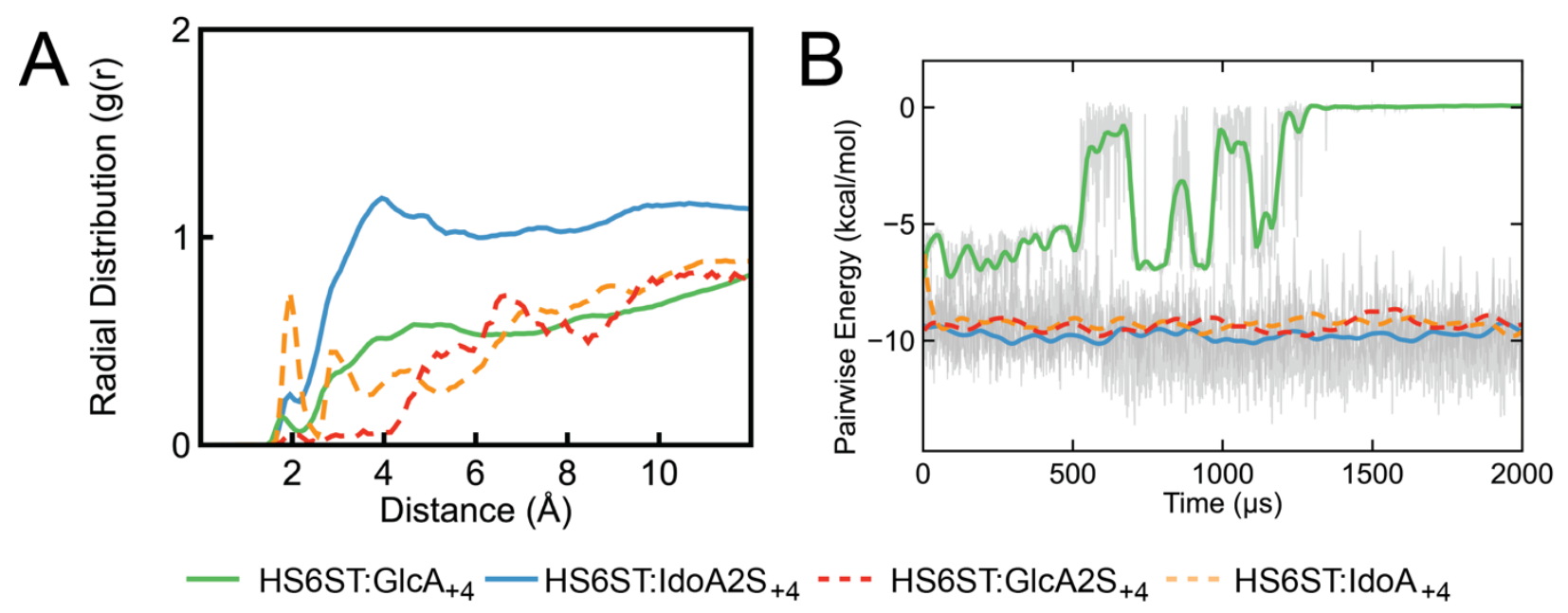

Figure S13. Molecular dynamics analysis of HS6ST. (A) Radial distribution of water around the catalytic histidine158 during HS6ST:IdoA+4 (blue), HS6ST:GlcA+4 (green, HS6ST:GlcA+4) simulations. (B) MM-GBSA pairwise decomposition between GlcNS and W158. 

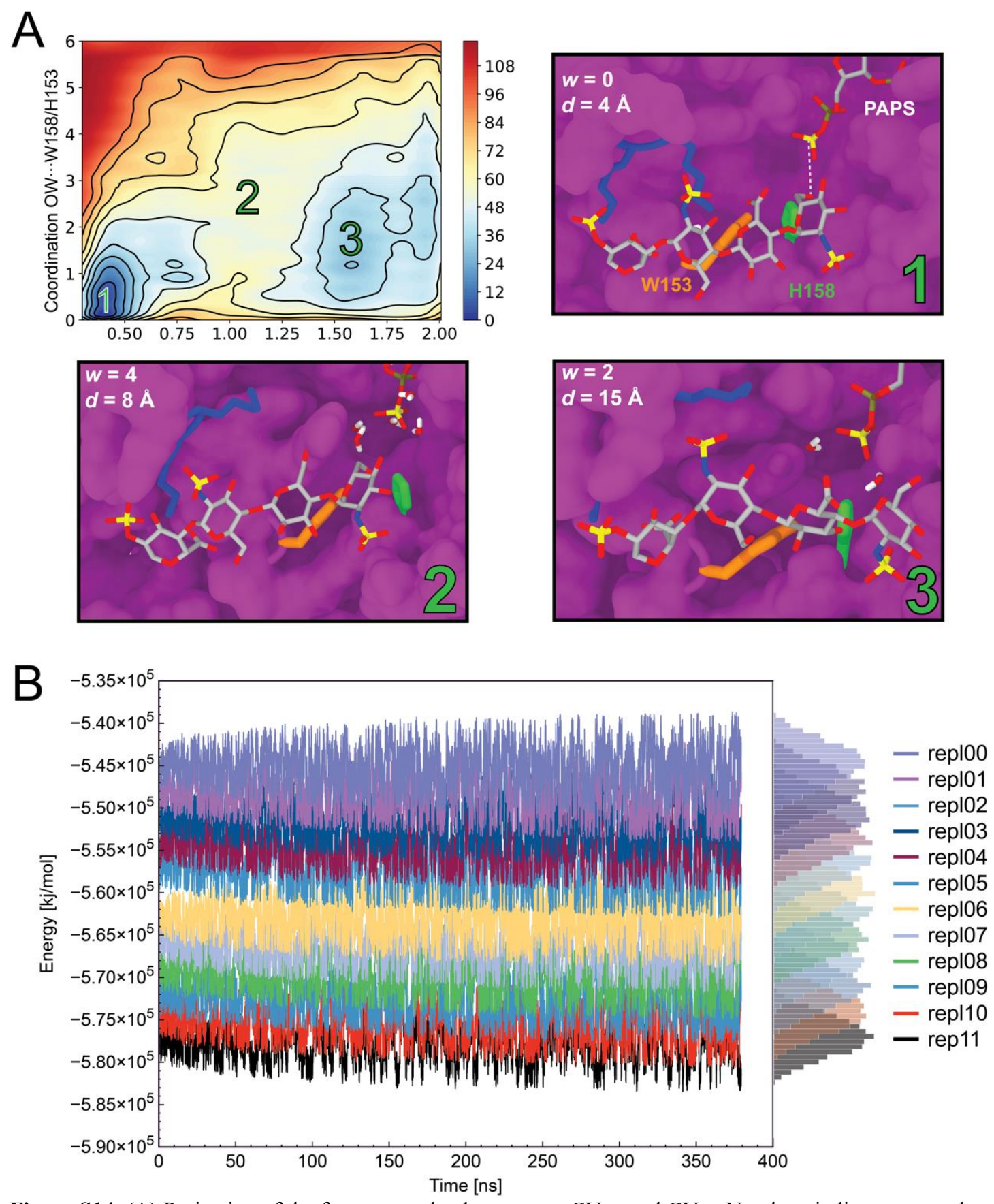

Figure S14. (A) Projection of the free-energy landscape onto $C_{\text {dist }}$ and $C_{\mathrm{w}}$, Numbers indicate most relevant metastable states 1 and 3, separated by a high energy barrier, 2. Energy is in units of kcal/mol, with contours drawn every $12 \mathrm{kcal} / \mathrm{mol}$. Representative conformation clustered from metastable states 1-3, and their water coordination (w) and $\mathrm{CV}_{\text {dist }}(\mathrm{d})$ for each state. Loop containing K131/K132 shown as blue sticks. (B) Energy distribution of the parallel tempering replicas (each represented by a different color) over water coordination metadynamics run biasing the energy for HS6ST in presence of PAPS and oligosaccharide substrate (IdoA2 $\mathrm{S}_{+4}$ ). 

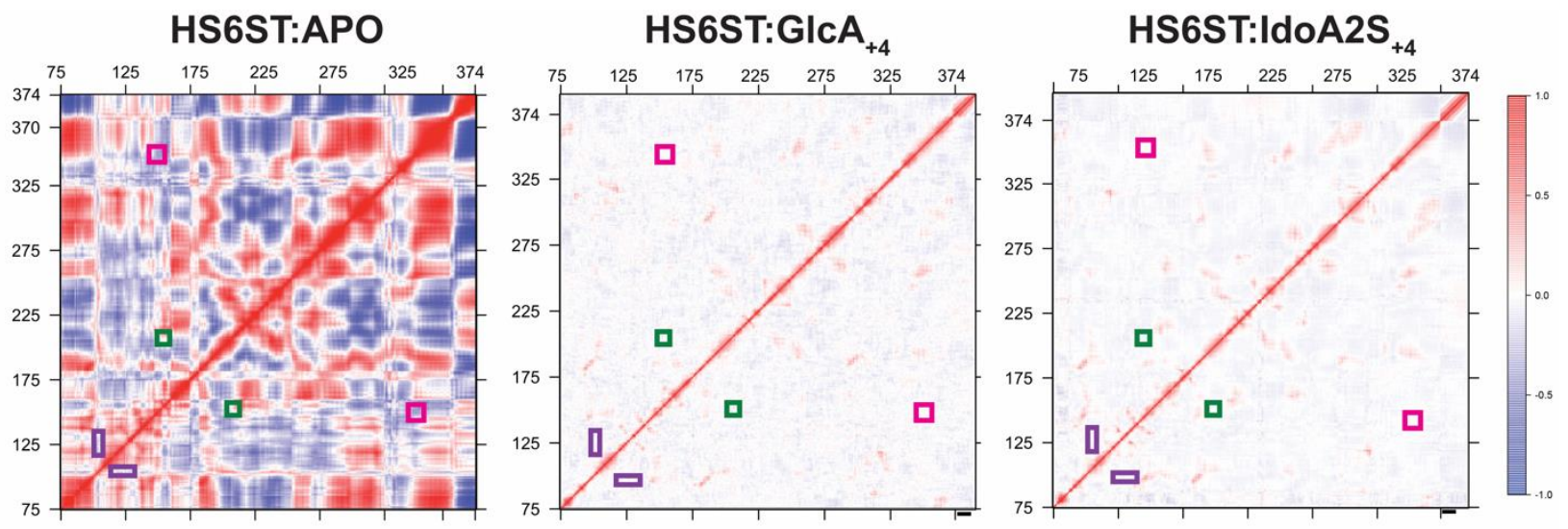

\section{HS6ST:GICA2S ${ }_{+4}$}

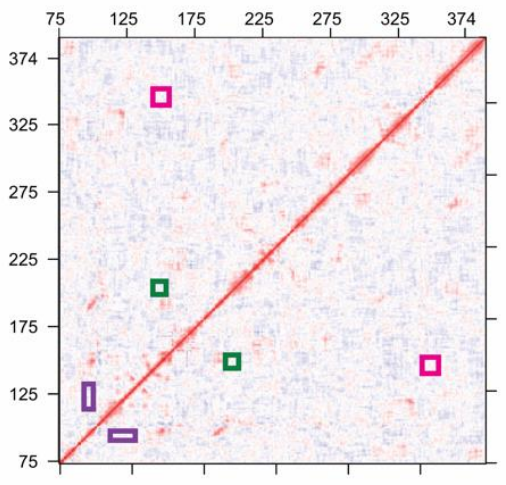

HS6ST:IdoA +4

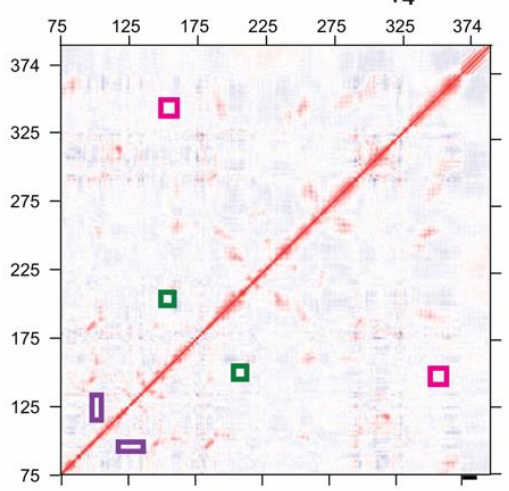

Figure S15. Cooperative motions underlying the functional dynamics in the apoprotein and sugar bound HS6STs. Perresidue Pearson's coefficients cross correlation matrix derived from the mass-weighted covariance matrix constructed over the last $1.2 \mu$ s of MD simulations for $\mathrm{C} \alpha$, sugar ring heavy atoms and PAPS nucleoside heavy atoms. CCs are comprised between -1 (anticorrelation, blue) and +1 (correlation, red). 

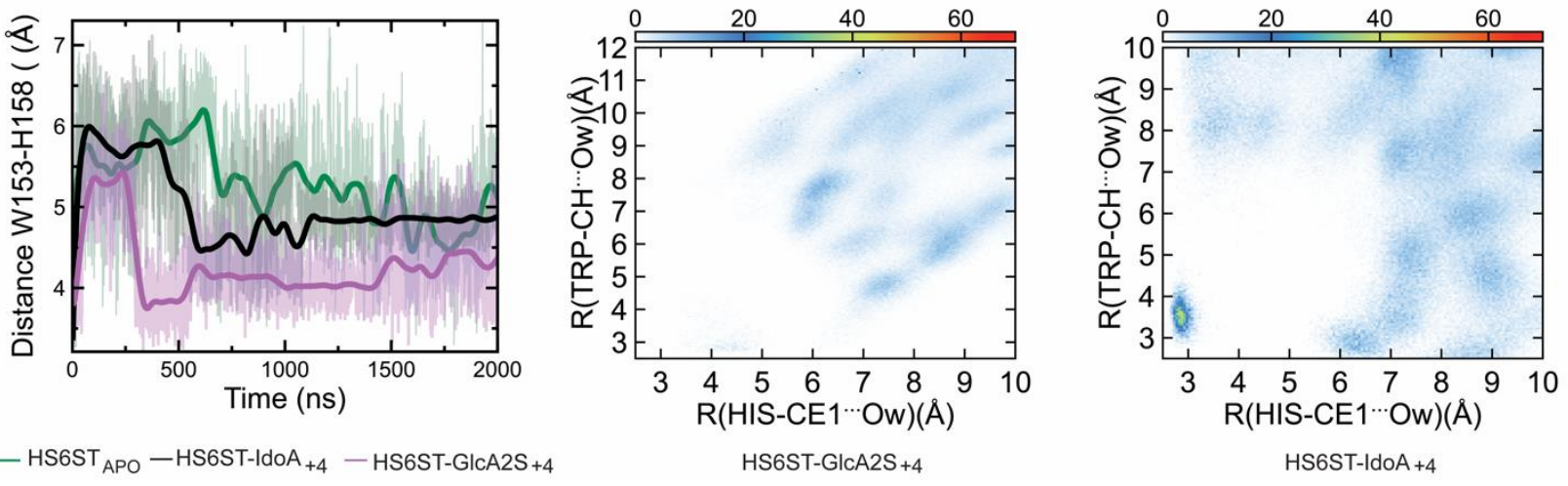

Figure S16. HS6ST substrate at position +4 influences HS6ST motility and active site hydration. Left panel, Distances between W153 and H158 during HS6ST IdoA +4 and GlcA2S +4 simulations. Center and right panels, 2D-RDF function for two closest atoms from W153 and H158 for both HS6ST:GlcA2S ${ }_{+4}$ and HS6ST:IdoA +4 simulations. Atoms are labeled according to AMBER force field nomenclature.

Discussion S4. Similar to what was observed for the HS2ST simulations, the HS6ST $\mathrm{T}_{\text {apo }}$ state displays the largest fluctuations (Figure S15, left panel) on its motions when compared with both HS6ST:GlcA +4 and HS6ST:IdoA2S +4 . Different to HS2ST, the HS6ST:GlcA +4 simulations show slightly less motion than the HS6ST:IdoA2S +4 (Figure S15, center and right panels). In the HS6ST:GlcA ${ }_{+4}$ simulations, the protein was unable to fully bind to the glycan in 1 out of 5 simulations, and the displacement of the glycan was observed. After losing main contacts with the K131/K132 loop, the substrate is unable to maintain interactions losing stability within the enzyme cleft. Additionally, as the polysaccharide substrate follows its biosynthetic pathway, the addition of $\mathrm{N}$ - and 2O- sulfates increases its hydrophilic nature. The K131/K132 pair forms hydrogen bonds with $\mathrm{GlcNS}_{+3}$ and IdoA2 $\mathrm{S}_{+4}$ cooperating in its binding. Mutating these positively charged residues, especially K132, radically decreases sulfation of substrates containing Ido $2 \mathrm{~S}_{+4},{ }^{22}$ as the enzyme is unable to displace the waters surrounding the sulfate. The lack of sulfation at position +4 impacts enzyme substrate interaction for GlcA more than IdoA, potentially due to the unmalleable nature of GlcA. There is one cluster of water molecules within $<4 \AA$ of W153 and H158 during HS6ST:IdoA +4 simulations (Figure S16, right panel). 
A
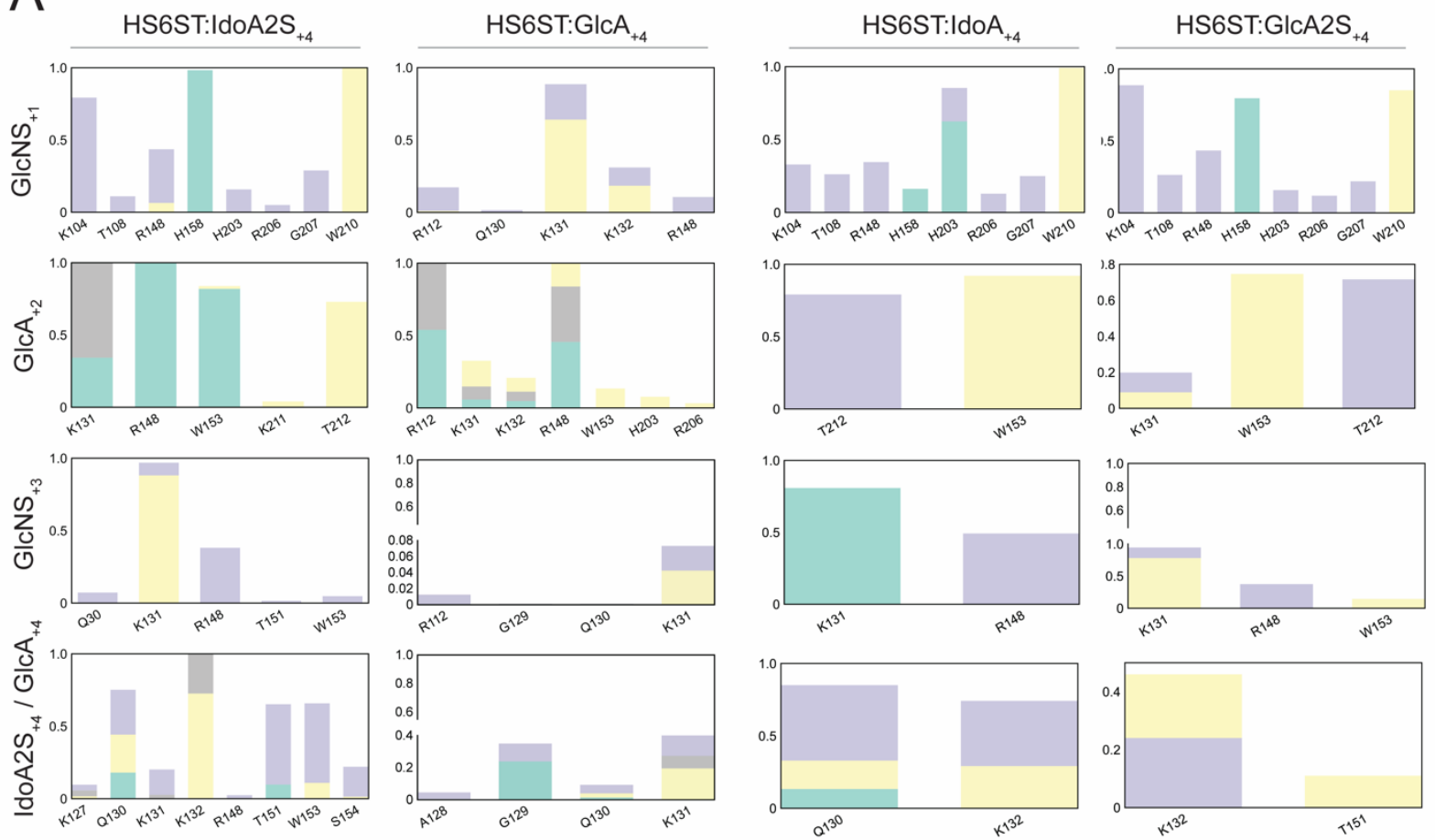

Water bridge Salt bridge Hydrogen-bond (donor) Hydrogen-bond (acceptor)

B

HS6ST:IdoA2S

HS6ST:GIcA +4
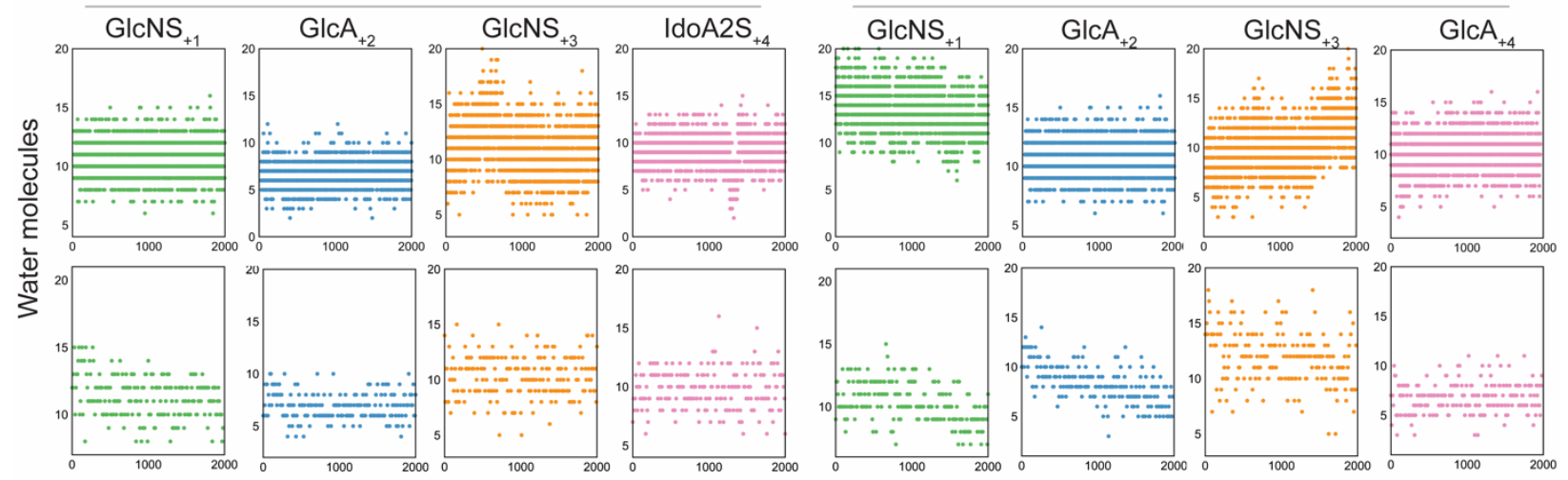

Time (ns)

Figure S17: (A) Enzyme ligand fingerprinting for HS6ST with IdoA2S $+4 / \mathrm{GlcA}_{+4} / \mathrm{IdoA}_{+4} / \mathrm{GlcA}_{2} \mathrm{~S}_{+4}$ as a substrate. Specific contacts between each composing monosaccharide are colored: Water bridge (purple), Salt bridge (gray), Hydrogen bonddonor (yellow) and Hydrogen bond-acceptor (green). (B) Water shell calculation for each HS6ST interfacing monosaccharide. Acceptor GlcNS; green, $\mathrm{GlcA}_{+2}$; blue, $\mathrm{GlcNS}_{+3}$; orange, $\mathrm{IdoA}_{+4} / \mathrm{GlcA}_{+4}$; pink. 

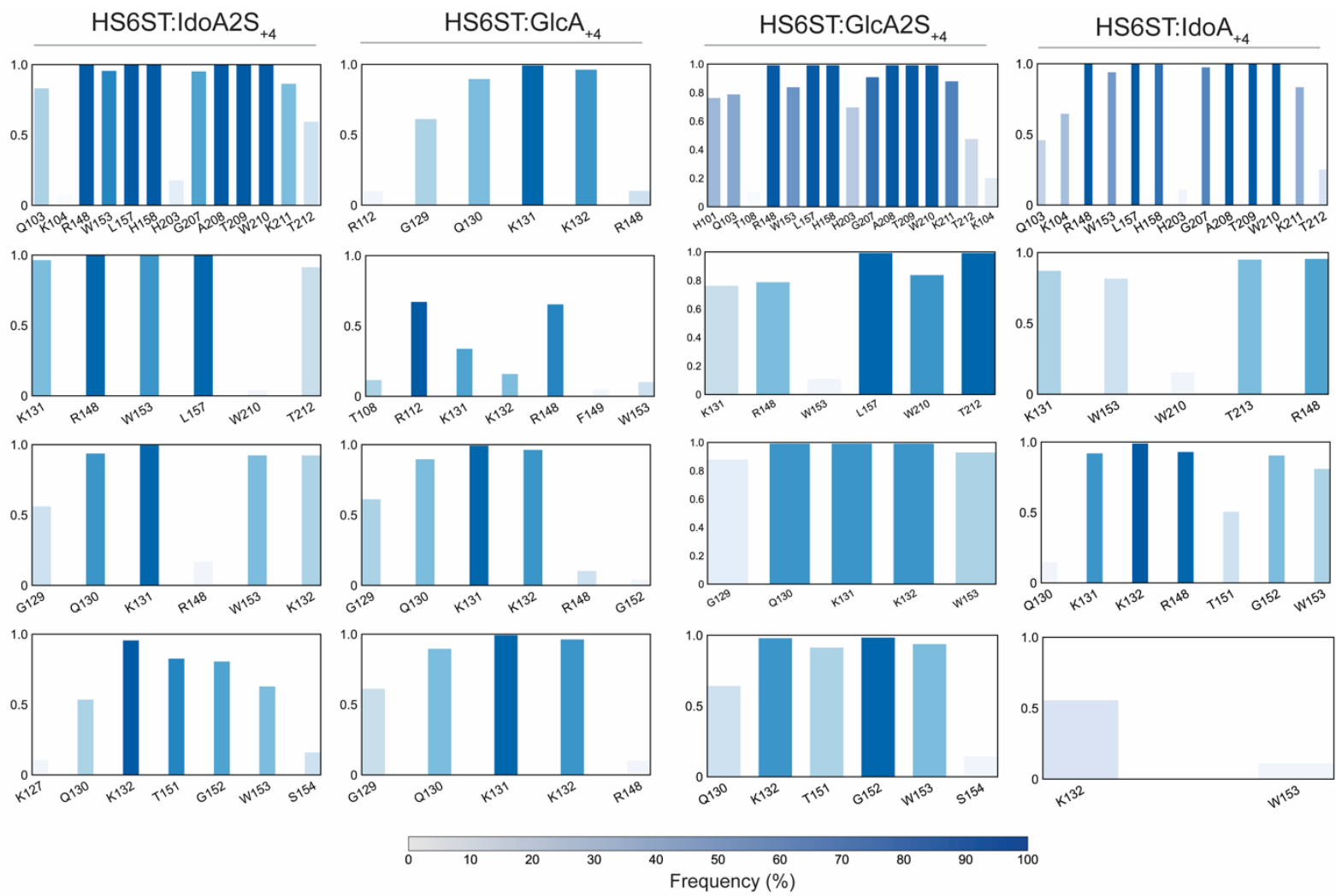

Figure S18: Number of distance-based contacts $<4 \AA$ for HS6ST with IdoA2 $\mathrm{S}_{+4} / \mathrm{GlcA}_{+4} / \mathrm{IdoA}_{+4} / \mathrm{GlcA}_{2} \mathrm{~S}_{+4}$ simulations. Graphs are colored by frequency from high $(100 \%$ - blue) to low $(0 \%$ - white).

Discussion S5. There is a significant reduction of contacts between $\mathrm{GlcNS}_{+3}$ and $\mathrm{K} 132$ during $\mathrm{HS}$ ST:GlcA $\mathrm{A}_{+4}$ simulations, when compared to IdoA2 $\mathrm{S}_{+4}$ simulations. During HS6ST:GlcA ${ }_{+4}$ simulations, most contacts K131 forms are hydrogen bonds with $\mathrm{GlcNS}_{+3}$ and salt bridges with $\mathrm{GlcA}_{+2}$. $\mathrm{GlcA}_{4+}$ still forms water bridges and hydrogen bonds with $\mathrm{K} 131$, but there are no contacts between the substrate and K132 (Figure S17 and S18). During HS6ST:IdoA ${ }_{+4}$ simulations the IdoA2 $\mathrm{S}_{4+}$ moiety forms both salt bridges and hydrogen bonds with K132, while K131 forms mostly water bridges. During the course of dynamics $0.08 \%$ of water bridges and $0.001 \%$ of hydrogens bonds were identified between GlcNS $_{+3}$ and $\mathrm{K} 132$ (Supporting Figure 17B). Consequently, this decrease in contacts between $\mathrm{GlcNS}_{+3} / \mathrm{GlcA}_{+4}$ and $\mathrm{K} 131 / \mathrm{K} 132$ observed when there is a GlcA at the +4 position causes a decrease of overall contacts between $\mathrm{GlcA}_{+2}$ and W153 (15\% hydrogen bonding), which persist during HS6ST:IdoA +4 simulations that present $89 \%$ hydrogens bonds (Figure S17).

By performing the interaction fingerprinting between each monosaccharide and their surrounding amino acids, we can picture the determinants that cause the polysaccharide substrate unbinding. While contacts between K131/K132 are the predominant contact for the reducing end $\mathrm{GlcA}_{+2}$ during the HS6ST:IdoA +4 simulations, HS6ST:GlcA $\mathrm{A}_{+4}$ has the reactive GlcNS $_{+1}$ as higher contact population, showing that the substrate is sliding away from the active site. Also, contacts between $\mathrm{H} 148$ and W210 with the acceptor GlcNS +1 are lost during the course of the HS6ST:GlcA $\mathrm{A}_{+4}$ simulations (Figure S18, second panel). The reason for the gradual loss of contacts between HS6ST and $\mathrm{GlcA}_{+4}$ during the course of the simulations can be explained by the lack of the negative charge which is present on the substrate during HS6ST:IdoA2 $\mathrm{S}_{+4}$ simulations. This causes the substrate to partially drift away from the binding cleft, with solely the non-reducing end remaining attached to W210. The loss of connections between the enzyme and substrate occurs concomitantly with an increase in the average number of water molecules surrounding $\mathrm{GlcNS}_{+1}$ and $\mathrm{GlcA}_{+2}$, this can be visualized after calculating the water network surrounding the substrate (Figure S17B). We found that the average water network was higher for HS6ST:GlcA $A_{+4}$ when compared to HS6ST:IdoA $A_{+4}$, specifically 13.3 vs 8.4 for $\mathrm{GlcNS}_{+1}$, and 11.2 vs 7.9 for $\mathrm{GlcA}_{+2}$. This lack of sulfation on $\mathrm{GlcA}_{+4}$ has an effect on the overall positioning of the K131/K132 loop, further explaining the enzyme preference for this sulfated moiety. 

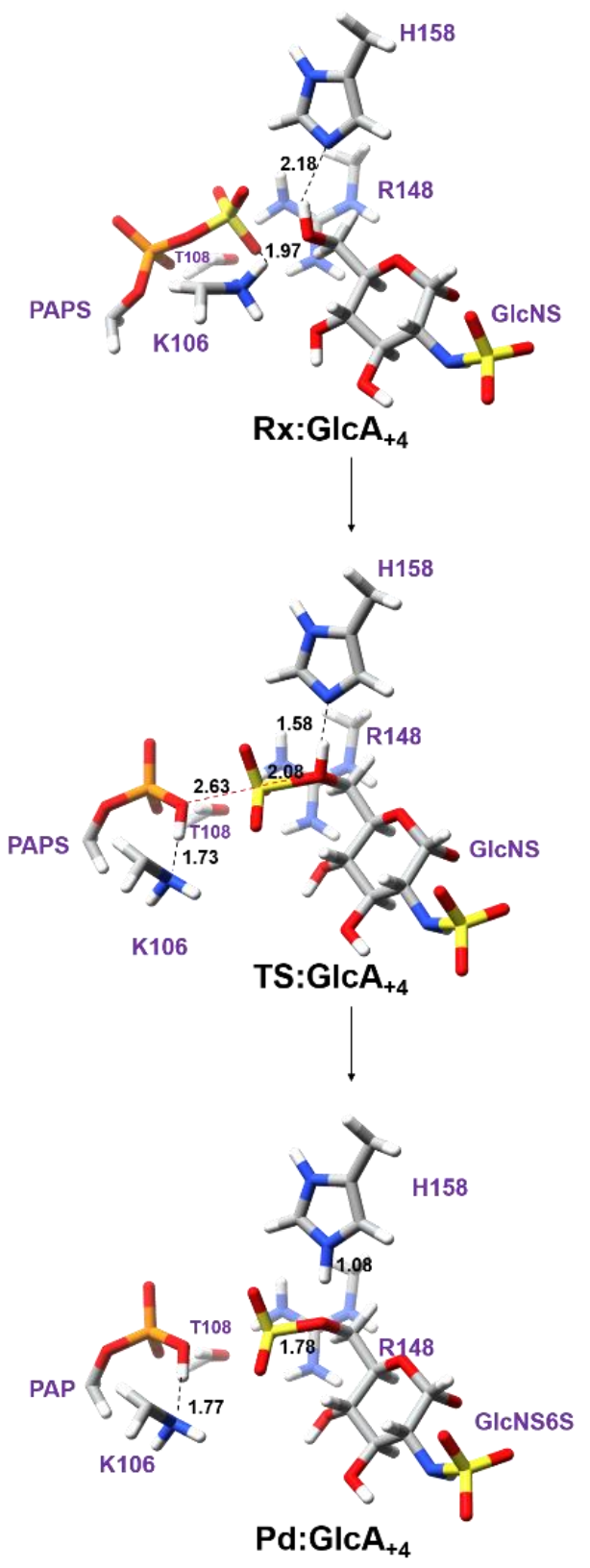

Figure S19. 3D-representation of the critical points obtained at the ONIOM[M06-2X/6-31+G*:ff14SB] level of theory for the catalytic mechanism of the sulfate transfer from PAPS to $\mathrm{GlcNS}_{+1}$ for HS6ST:GlcA +4 . 
Discussion S6. At the reactant complex Rx:GlcA+4 (Figure S19, top panel) the polar sidechain of T108 interacts with the phosphate moiety of PAPS $(1.59 \AA)$ while R148 anchors threonine by a strong hydrogen bond engaged by the guanidinium group (1.73 $\AA$ ). Interestingly, R148 does not interact with the sugar, as much as it happened in HS2ST, where one of the two arginines of the HL were responsible for anchoring the uronic acid. The positively charged group of K104 interacts with C5$\mathrm{OH}, \mathrm{C} 6-\mathrm{OH}$ and the sulfo group $(2.06 \AA, 1.77 \AA$ and $1.97 \AA$ respectively). The hydrogen of C6-OH generates a polar interaction with the nitrogen of H158 (2.18 $\AA$ ). The reaction proceeds overcoming the TS: GlcA+4 depicted in Figure S20 (middle panel), where the sulfur atom of the $\mathrm{SO}_{3}{ }^{-}$group is $2.63 \AA$ away from the phosphate group, and $2.08 \AA$ from to the nucleophile (C6-OH). At the transition state, $\mathrm{C} 6 \mathrm{O}$ is still protonated, with a distance for $\mathrm{N} \cdots \mathrm{H}-\mathrm{O} 6$ of $1.58 \AA$ while $\mathrm{K} 104$ is deprotonated and proton has already migrated from the $\mathrm{NH}_{3}{ }^{+}$to the phosphate group. Overcoming the transition state, the system collapses into the product complex Pd:GlcA+4 , where the $\mathrm{SO}_{3}-$ OC6 bond is completely formed (1.78 $\AA$ ), the histidine is fully protonated (N-H being $1.08 \AA$ ) and lysine is present in its neutral form. Also, as reported in Figure S19 (bottom panel), R148 interacts with T108 via a strong hydrogen bond (1.52 $\AA$ ). 

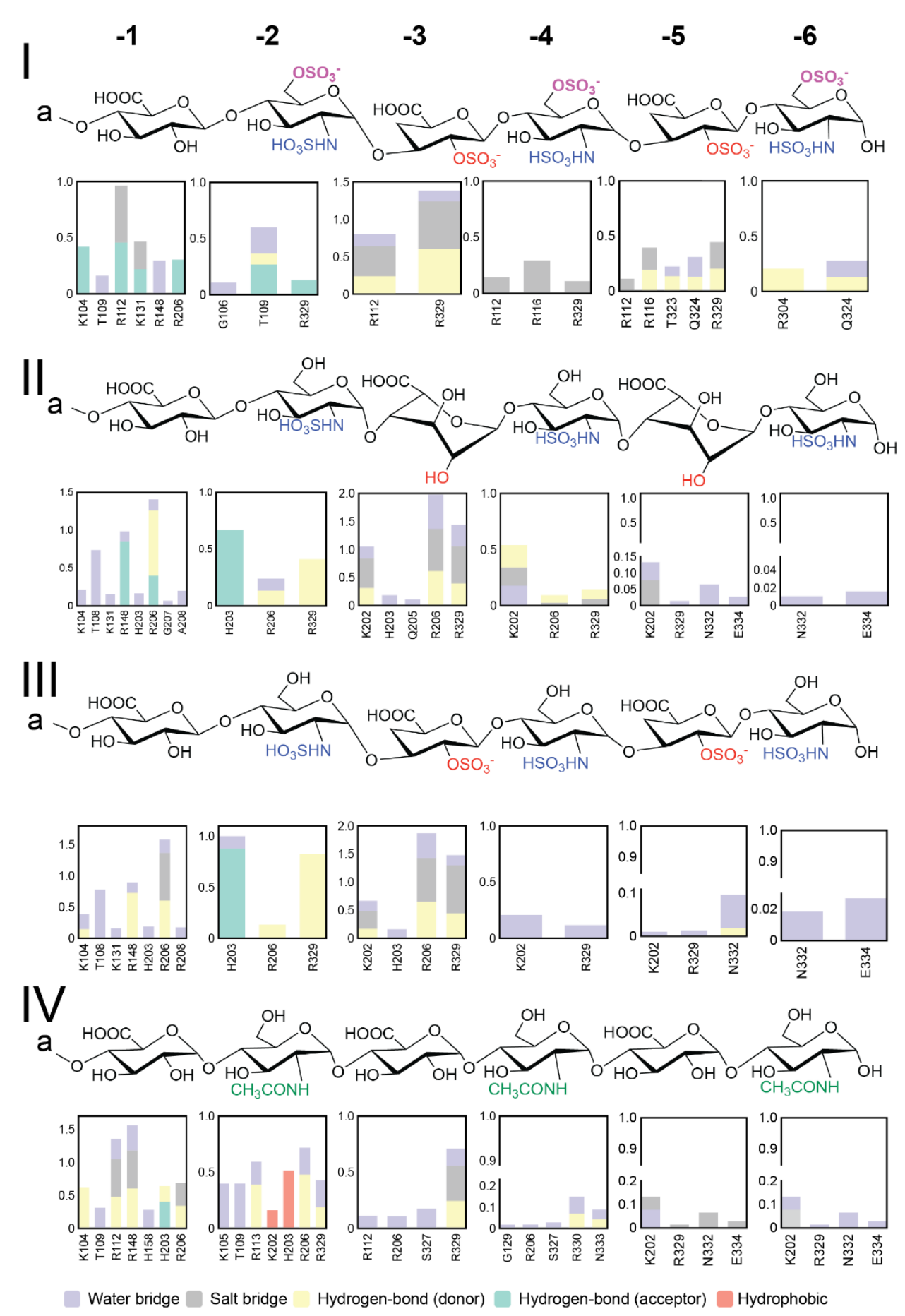

Figure S20. Enzyme-ligand fingerprinting for HS6ST:Ido2S $\mathrm{S}_{+4}$ towards non-reducing end extensions. (I) IdoA2S-GlcNS, (II) IdoA-GlcNS, (III) GlcA2S-GlcNS, (IV) GlcA-GlcNAc. Lowercase a represents acceptor GlcNS. Bar charts under each unit represent interactions as measure during molecular dynamics simulations. 

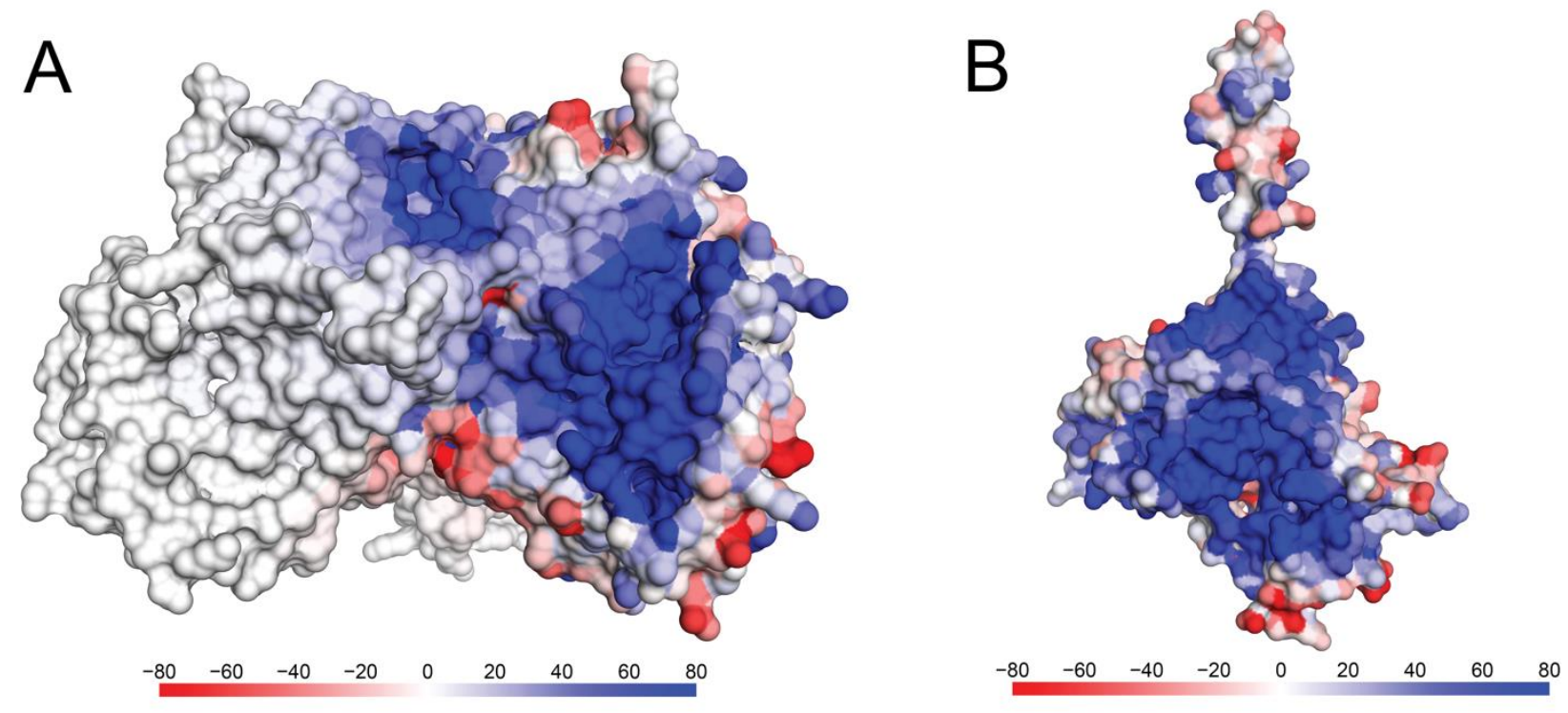

Figure S21: The electrostatic surface potential (generated with APBS ${ }^{1}$ of HS2ST (A) and HSST (B). Calculated solvation free energies on the surface of the binding interfaces of HS2ST (C) and HS6ST (D) as a measure of their hydrophobicity. Right table shows per-residue average hydrophobicity at each APO ST. Table color range from more hydrophobic, i.e., higher score on the hydrophobicity scale (purple), to more hydrophilic, i.e., lower score on the hydrophobicity scale (green). Asterisk denotes C-terminal residues from adjacent monomer. 


\section{References}

1. Jurrus, E.; Engel, D.; Star, K.; Monson, K.; Brandi, J.; Felberg, L. E.; Brookes, D. H.; Wilson, L.; Chen, J.; Liles, K.; Chun, M.; Li, P.; Gohara, D. W.; Dolinsky, T.; Konecny, R.; Koes, D. R.; Nielsen, J. E.; Head-Gordon, T.; Geng, W.; Krasny, R.; Wei, G. W.; Holst, M. J.; McCammon, J. A.; Baker, N. A., Improvements to the APBS biomolecular solvation software suite. Protein Sci 2018, 27 (1), 112-128.

2. Woods Group. (2005-2019) GLYCAM Web. Complex Carbohydrate Research Center, University of Georgia, Athens, GA. (http://glycam.org).

3. Liu, C.; Sheng, J.; Krahn, J. M.; Perera, L.; Xu, Y.; Hsieh, P. H.; Dou, W.; Liu, J.; Pedersen, L. C., Molecular mechanism of substrate specificity for heparan sulfate 2-Osulfotransferase. J Biol Chem 2014, 289 (19), 13407-18.

4. Xu, D.; Song, D.; Pedersen, L. C.; Liu, J., Mutational study of heparan sulfate 2-Osulfotransferase and chondroitin sulfate 2-O-sulfotransferase. J Biol Chem 2007, 282 (11), 835667.

5. Mark, P.; Nilsson, L., Structure and Dynamics of the TIP3P, SPC, and SPC/E Water Models at 298 K. The Journal of Physical Chemistry A 2001, 105 (43), 9954-9960.

6. Anandakrishnan, R.; Aguilar, B.; Onufriev, A. V., H++ 3.0: automating pK prediction and the preparation of biomolecular structures for atomistic molecular modeling and simulations. Nucleic Acids Res 2012, 40 (Web Server issue), W537-41.

7. Loncharich, R. J.; Brooks, B. R.; Pastor, R. W., Langevin dynamics of peptides: the frictional dependence of isomerization rates of $\mathrm{N}$-acetylalanyl-N'-methylamide. Biopolymers 1992, 32 (5), 523-35.

8. Berendsen, H. J. C.; Postma, J. P. M.; van Gunsteren, W. F.; DiNola, A.; Haak, J. R., Molecular dynamics with coupling to an external bath. The Journal of Chemical Physics 1984, 81 (8), 3684-3690.

9. Miyamoto, S.; Kollman, P. A., Settle: An analytical version of the SHAKE and RATTLE algorithm for rigid water models. Journal of Computational Chemistry 1992, 13 (8), 952-962.

10. Ryckaert, J.-P.; Ciccotti, G.; Berendsen, H., Numerical-Integration of Cartesian Equations of Motion of a System with Constraints - Molecular-Dynamics of N-Alkanes. Journal of Computational Physics 1977, 23, 327-341.

11. D.A. Case, R. M. B., D.S. Cerutti, T.E. Cheatham, III, T.A. Darden, R.E. Duke, T.J. Giese, H. Gohlke, A.W. Goetz, N. Homeyer, S. Izadi, P. Janowski, J. Kaus, A. Kovalenko, T.S. Lee, S. LeGrand, P. Li, C. Lin, T. Luchko, R. Luo, B. Madej, D. Mermelstein, K.M. Merz, G. Monard, H. Nguyen, H.T. Nguyen, I. Omelyan, A. Onufriev, D.R. Roe, A. Roitberg, C. Sagui, C.L. Simmerling, W.M. Botello-Smith, J. Swails, R.C. Walker, J. Wang, R.M. Wolf, X. Wu, L. Xiao and P.A. Kollman AMBER 2016, University of California, San Francisco, 2016.

12. Le Grand, S.; Götz, A. W.; Walker, R. C., SPFP: Speed without compromise-A mixed precision model for GPU accelerated molecular dynamics simulations. Computer Physics Communications 2013, 184 (2), 374-380.

13. Hou, T.; Wang, J.; Li, Y.; Wang, W., Assessing the performance of the MM/PBSA and MM/GBSA methods. 1 . The accuracy of binding free energy calculations based on molecular dynamics simulations. J Chem Inf Model 2011, 51 (1), 69-82. 
14. Genheden, S.; Ryde, U., The MM/PBSA and MM/GBSA methods to estimate ligandbinding affinities. Expert Opin Drug Discov 2015, 10 (5), 449-61.

15. Sun, H.; Duan, L.; Chen, F.; Liu, H.; Wang, Z.; Pan, P.; Zhu, F.; Zhang, J. Z. H.; Hou, T., Assessing the performance of MM/PBSA and MM/GBSA methods. 7. Entropy effects on the performance of end-point binding free energy calculation approaches. Phys Chem Chem Phys 2018, 20 (21), 14450-14460.

16. D.A. Case, I. Y. B.-S., S.R. Brozell, D.S. Cerutti, T.E. Cheatham, III, V.W.D. Cruzeiro, T.A. Darden, R.E. Duke, D. Ghoreishi, M.K. Gilson, H. Gohlke, A.W. Goetz, D. Greene, R Harris, N. Homeyer, S. Izadi, A. Kovalenko, T. Kurtzman, T.S. Lee, S. LeGrand, P. Li, C. Lin, J. Liu, T. Luchko, R. Luo, D.J. Mermelstein, K.M. Merz, Y. Miao, G. Monard, C. Nguyen, H. Nguyen, I. Omelyan, A. Onufriev, F. Pan, R. Qi, D.R. Roe, A. Roitberg, C. Sagui, S. SchottVerdugo, J. Shen, C.L. Simmerling, J. Smith, R. Salomon-Ferrer, J. Swails, R.C. Walker, J. Wang, H. Wei, R.M. Wolf, X. Wu, L. Xiao, D.M. York and P.A. Kollman AMBER 2016, University of California, San Francisco., 2016.

17. Case, D. A.; Cheatham, T. E., 3rd; Darden, T.; Gohlke, H.; Luo, R.; Merz, K. M., Jr.; Onufriev, A.; Simmerling, C.; Wang, B.; Woods, R. J., The Amber biomolecular simulation programs. J Comput Chem 2005, 26 (16), 1668-88.

18. Robert, X.; Gouet, P., Deciphering key features in protein structures with the new ENDscript server. Nucleic Acids Res 2014, 42 (Web Server issue), W320-4.

19. Li, K.; Bethea, H. N.; Liu, J., Using engineered 2-O-sulfotransferase to determine the activity of heparan sulfate C5-epimerase and its mutants. J Biol Chem 2010, 285 (15), 11106-13. 20. Chen, J.; Jones, C. L.; Liu, J., Using an enzymatic combinatorial approach to identify anticoagulant heparan sulfate structures. Chem Biol 2007, 14 (9), 986-93.

21. Carnachan, S. M.; Hinkley, S. F. R., Heparan Sulfate Identification and Characterisation: Method II. Enzymatic Depolymerisation and SAX-HPLC Analysis to Determine Disaccharide Composition. Bio-protocol 2017, 7 (7), e2197.

22. Xu, Y.; Moon, A. F.; Xu, S.; Krahn, J. M.; Liu, J.; Pedersen, L. C., Structure Based Substrate Specificity Analysis of Heparan Sulfate 6-O-Sulfotransferases. ACS Chem Biol 2017, $12(1), 73-82$.

23. consortium, P., Promoting transparency and reproducibility in enhanced molecular simulations. Nat Methods 2019, 16 (8), 670-673.

24. Laio, A.; Parrinello, M., Escaping free-energy minima. Proc Natl Acad Sci U S A 2002, 99 (20), 12562-6.

25. Barducci, A.; Bonomi, M.; Parrinello, M., Metadynamics. WIREs Computational Molecular Science 2011, 1 (5), 826-843.

26. Bonomi, M.; Branduardi, D.; Bussi, G.; Camilloni, C.; Provasi, D.; Raiteri, P.; Donadio, D.; Marinelli, F.; Pietrucci, F.; Broglia, R. A.; Parrinello, M., PLUMED: A portable plugin for free-energy calculations with molecular dynamics. Computer Physics Communications 2009, 180 (10), 1961-1972.

27. Cremer, D.; Pople, J. A., General definition of ring puckering coordinates. Journal of the American Chemical Society 1975, 97 (6), 1354-1358.

28. Vreven, T.; Byun, K. S.; Komaromi, I.; Dapprich, S.; Montgomery, J. A.; Morokuma, K.; Frisch, M. J., Combining Quantum Mechanics Methods with Molecular Mechanics Methods in ONIOM. J Chem Theory Comput 2006, 2 (3), 815-26.

29. Dapprich, S.; Komáromi, I.; Byun, K. S.; Morokuma, K.; Frisch, M. J., A new ONIOM implementation in Gaussian98. Part I. The calculation of energies, gradients, vibrational 
frequencies and electric field derivatives1Dedicated to Professor Keiji Morokuma in celebration of his 65th birthday.1. Journal of Molecular Structure: THEOCHEM 1999, 461-462, 1-21.

30. Frisch, M. J.; Trucks, G. W.; Schlegel, H. B.; Scuseria, G. E.; Robb, M. A.;

Cheeseman, J. R.; Scalmani, G.; Barone, V.; Petersson, G. A.; Nakatsuji, H.; Li, X.; Caricato, M.; Marenich, A. V.; Bloino, J.; Janesko, B. G.; Gomperts, R.; Mennucci, B.; Hratchian, H.

P.; Ortiz, J. V.; Izmaylov, A. F.; Sonnenberg, J. L.; Williams; Ding, F.; Lipparini, F.; Egidi,

F.; Goings, J.; Peng, B.; Petrone, A.; Henderson, T.; Ranasinghe, D.; Zakrzewski, V. G.;

Gao, J.; Rega, N.; Zheng, G.; Liang, W.; Hada, M.; Ehara, M.; Toyota, K.; Fukuda, R.;

Hasegawa, J.; Ishida, M.; Nakajima, T.; Honda, Y.; Kitao, O.; Nakai, H.; Vreven, T.;

Throssell, K.; Montgomery Jr., J. A.; Peralta, J. E.; Ogliaro, F.; Bearpark, M. J.; Heyd, J. J.;

Brothers, E. N.; Kudin, K. N.; Staroverov, V. N.; Keith, T. A.; Kobayashi, R.; Normand, J.;

Raghavachari, K.; Rendell, A. P.; Burant, J. C.; Iyengar, S. S.; Tomasi, J.; Cossi, M.; Millam, J. M.; Klene, M.; Adamo, C.; Cammi, R.; Ochterski, J. W.; Martin, R. L.; Morokuma, K.; Farkas, O.; Foresman, J. B.; Fox, D. J. Gaussian 16 Rev. C.01, Wallingford, CT, 2016.

31. Chung, L. W.; Sameera, W. M.; Ramozzi, R.; Page, A. J.; Hatanaka, M.; Petrova, G.

P.; Harris, T. V.; Li, X.; Ke, Z.; Liu, F.; Li, H. B.; Ding, L.; Morokuma, K., The ONIOM

Method and Its Applications. Chem Rev 2015, 115 (12), 5678-796.

32. Neese, F., Software update: the ORCA program system, version 4.0. WIREs

Computational Molecular Science 2018, 8 (1), e1327.

33. Grimme, S.; Ehrlich, S.; Goerigk, L., Effect of the damping function in dispersion corrected density functional theory. J Comput Chem 2011, 32 (7), 1456-65.

34. Kovacevic, B.; Baric, D.; Babic, D.; Bilic, L.; Hanzevacki, M.; Sandala, G. M.; Radom, L.; Smith, D. M., Computational Tale of Two Enzymes: Glycerol Dehydration With or Without B12. J Am Chem Soc 2018, 140 (27), 8487-8496.

35. De Raffele, D.; Martí, S.; Moliner, V., QM/MM Theoretical Studies of a de Novo RetroAldolase Design. ACS Catalysis 2019, 9 (3), 2482-2492.

36. Campesato, L.; Marforio, T. D.; Giacinto, P.; Calvaresi, M.; Bottoni, A., A Full QM Computational Study of the Catalytic Mechanism of alpha-1,4-Glucan Lyases. Chemphyschem 2018, 19 (12), 1514-1521.

37. Marforio, T. D.; Giacinto, P.; Bottoni, A.; Calvaresi, M., Computational Evidence for the Catalytic Mechanism of Tyrosylprotein Sulfotransferases: A Density Functional Theory Investigation. Biochemistry 2015, 54 (28), 4404-4410.

38. Świderek, K.; Tuñón, I.; Williams, I. H.; Moliner, V., Insights on the Origin of Catalysis on Glycine N-Methyltransferase from Computational Modeling. Journal of the American Chemical Society 2018, 140 (12), 4327-4334.

39. Humphrey, W.; Dalke, A.; Schulten, K., VMD: Visual molecular dynamics. Journal of Molecular Graphics 1996, 14 (1), 33-38.

40. Pettersen, E. F.; Goddard, T. D.; Huang, C. C.; Couch, G. S.; Greenblatt, D. M.;

Meng, E. C.; Ferrin, T. E., UCSF Chimera-A visualization system for exploratory research and analysis. Journal of Computational Chemistry 2004, 25 (13), 1605-1612.

41. Schrodinger, LLC, The PyMOL Molecular Graphics System, Version 1.8. 2015.

42. Makeneni, S.; Foley, B. L.; Woods, R. J., BFMP: a method for discretizing and visualizing pyranose conformations. J Chem Inf Model 2014, 54 (10), 2744-50.

43. Roe, D. R.; Cheatham, T. E., 3rd, PTRAJ and CPPTRAJ: Software for Processing and Analysis of Molecular Dynamics Trajectory Data. J Chem Theory Comput 2013, 9 (7), 3084-95. 
44. Kokh, D. B.; Doser, B.; Richter, S.; Ormersbach, F.; Cheng, X.; Wade, R. C., A workflow for exploring ligand dissociation from a macromolecule: Efficient random acceleration molecular dynamics simulation and interaction fingerprint analysis of ligand trajectories. $J$ Chem Phys 2020, 153 (12), 125102.

45. Abel, R.; Salam, N. K.; Shelley, J.; Farid, R.; Friesner, R. A.; Sherman, W., Contribution of explicit solvent effects to the binding affinity of small-molecule inhibitors in blood coagulation factor serine proteases. ChemMedChem 2011, 6 (6), 1049-66.

46. Nguyen, C. N.; Cruz, A.; Gilson, M. K.; Kurtzman, T., Thermodynamics of Water in an Enzyme Active Site: Grid-Based Hydration Analysis of Coagulation Factor Xa. J Chem Theory Comput 2014, 10 (7), 2769-2780.

47. Ramsey, S.; Nguyen, C.; Salomon-Ferrer, R.; Walker, R. C.; Gilson, M. K.; Kurtzman, T., Solvation thermodynamic mapping of molecular surfaces in AmberTools: GIST. J Comput Chem 2016, 37 (21), 2029-37.

48. Skjaerven, L.; Yao, X. Q.; Scarabelli, G.; Grant, B. J., Integrating protein structural dynamics and evolutionary analysis with Bio3D. BMC Bioinformatics 2014, 15, 399.

49. Stankovic, I. M.; Blagojevic Filipovic, J. P.; Zaric, S. D., Carbohydrate - Protein aromatic ring interactions beyond $\mathrm{CH} /$ pi interactions: A Protein Data Bank survey and quantum chemical calculations. Int J Biol Macromol 2020, 157, 1-9.

50. Montalvillo-Jimenez, L.; Santana, A. G.; Corzana, F.; Jimenez-Oses, G.; JimenezBarbero, J.; Gomez, A. M.; Asensio, J. L., Impact of Aromatic Stacking on Glycoside Reactivity: Balancing $\mathrm{CH} /$ pi and Cation/pi Interactions for the Stabilization of GlycosylOxocarbenium Ions. J Am Chem Soc 2019, 141 (34), 13372-13384.

51. Hudson, K. L.; Bartlett, G. J.; Diehl, R. C.; Agirre, J.; Gallagher, T.; Kiessling, L. L.; Woolfson, D. N., Carbohydrate-Aromatic Interactions in Proteins. J Am Chem Soc 2015, 137 (48), 15152-60.

52. Wang, J.; Yao, L., Dissecting C-Hpi and N-Hpi Interactions in Two Proteins Using a Combined Experimental and Computational Approach. Sci Rep 2019, 9 (1), 20149. 